MATHEMATICS OF COMPUTATION

Volume 76, Number 258, April 2007, Pages 539-571

S 0025-5718(06)01915-6

Article electronically published on November 20, 2006

\title{
ANALYSIS OF FINITE ELEMENT APPROXIMATIONS OF A PHASE FIELD MODEL FOR TWO-PHASE FLUIDS
}

\author{
XIAOBING FENG, YINNIAN HE, AND CHUN LIU
}

\begin{abstract}
This paper studies a phase field model for the mixture of two immiscible and incompressible fluids. The model is described by a nonlinear parabolic system consisting of the nonstationary Stokes equations coupled with the Allen-Cahn equation through an extra phase induced stress term in the Stokes equations and a fluid induced transport term in the Allen-Cahn equation. Both semi-discrete and fully discrete finite element methods are developed for approximating the parabolic system. It is shown that the proposed numerical methods satisfy a discrete energy law which mimics the basic energy law for the phase field model. Error estimates are derived for the semi-discrete method, and the convergence to the phase field model and to its sharp interface limiting model are established for the fully discrete finite element method by making use of the discrete energy law. Numerical experiments are also presented to validate the theory and to show the effectiveness of the combined phase field and finite element approach.
\end{abstract}

\section{INTRODUCTION}

In this paper we propose and analyze some semi-discrete and fully discrete finite element methods for the following phase field model for two immiscible and incompressible fluids with comparable densities (which are taken to be 1) and viscosities $\nu>0$ (cf. 24]):

$$
\begin{array}{rlrl}
u_{t}-\nu \Delta u+\nabla p+\lambda \operatorname{div}(\nabla \phi \otimes \nabla \phi) & =g & & \text { in } \Omega_{T}:=\Omega \times(0, T], T>0, \\
\phi_{t}+u \cdot \nabla \phi-\gamma\left(\Delta \phi-\frac{1}{\varepsilon^{2}} f(\phi)\right)=0 & & \text { in } \Omega_{T}, \\
\operatorname{div} u=0 & & \text { in } \Omega_{T},
\end{array}
$$

with the following initial and boundary conditions:

$$
\begin{array}{lll}
u(x, 0)=u_{0}^{\varepsilon}(x), & \phi(x, 0)=\phi_{0}^{\varepsilon}(x), & \forall x \in \Omega, \\
u(x, t)=0, & \frac{\partial \phi(x, t)}{\partial n}=0, & \forall(x, t) \in \partial \Omega_{T}:=\partial \Omega \times(0, T] .
\end{array}
$$

Note that we have suppressed the superscript $\varepsilon$ in $\left(u^{\varepsilon}, \phi^{\varepsilon}, p^{\varepsilon}\right)$ for notational brevity. Here $\Omega \subset \mathbf{R}^{d}(d=2,3)$ is bounded domain with $C^{2}$ boundary $\partial \Omega$. The vector $u(x, t) \in \mathbf{R}^{d}$ and the scalar $p(x, t) \in \mathbf{R}$ denote the velocity and the pressure of the

Received by the editor April 28, 2005 and, in revised form, August 10, 2005.

2000 Mathematics Subject Classification. Primary 65M60, 35K55, 76D05.

Key words and phrases. Two phase fluids, phase field model, Allen-Cahn equation, Stokes equations, finite element methods.

The work of the first author was partially supported by the NSF grant DMS-0410266.

The work of the second author was partially supported by the NSF of China grant \#10671154. 
fluid mixture at the space time point $(x, t)$, respectively. The scalar function $\phi$ is called a phase function and is used to indicate the fluid phases. $\phi$ assumes distinct values in the bulk phases away from a thin layer (called the interfacial region) over which $\phi$ varies smoothly, and the interface itself can be associated with the zero level set $\{\phi=0\}$ of $\phi$. Also in the model, $f(\phi)=F^{\prime}(\phi)$ and $F(\phi)=\frac{1}{4}\left(\phi^{2}-1\right)^{2}$. The positive constants $\lambda, \gamma$ and $\varepsilon$ are the surface tension, the elastic relaxation time, and the capillary width (width of the interficial layer), respectively. $\nabla \phi \otimes \nabla \phi$ stands for the $d \times d$ rank-one matrix $(\nabla \phi)^{T} \nabla \phi$. It should be noted that $\varepsilon<<1$. Equation (1.1) without the stress term $\lambda \operatorname{div}(\nabla \phi \otimes \nabla \phi)$ is the nonstationary Stokes equation 27, and equation (1.2) without the convection term $u \cdot \nabla \phi$ is the Allen-Cahn (scalar Ginzburg-Landau) equation [15, 26. We remark that the original phase field model for two immiscible fluids proposed in 24] couples the Navier-Stokes equations with the Allen-Cahn equation. To avoid some technicalities and to present the idea on how to handle the coupling terms, in this paper we only consider the simplified model (1.1)-(1.5) and shall address the full model in a forthcoming paper.

Interfacial dynamics in the mixture of different fluids, solids or gas has been one of the fundamental issues in hydrodynamics and materials science. It plays an increasingly important role in many current scientific, engineering, and industrial applications (cf. [5, 11] and the references therein). In the classical approaches, the interface is usually considered as a free curve/surface that evolves in time along with fluid. The movement of the interface at each time is determined by a set of interfacial balance conditions. In the case of two immiscible incompressible fluids, the dynamics of the fluid mixture is described by the following coupled nonstationary Stokes equations:

$$
\begin{aligned}
u_{t}-\nu \Delta u+\nabla p=g & \text { in } \Omega_{T} \backslash \Gamma_{t}, \\
\operatorname{div} u=0 & \text { in } \Omega_{T} \backslash \Gamma_{t}, \\
{[(\nu D(u)-p I) n]=\alpha \kappa n } & \text { on } \Gamma_{t}, \\
{[u]=0 } & \text { on } \Gamma_{t},
\end{aligned}
$$

with the following initial and boundary conditions:

$$
\begin{array}{ll}
u(x, 0)=u_{0}^{\varepsilon}(x), & \forall x \in \Omega \backslash \Gamma_{0}, \\
u(x, t)=0, & \forall(x, t) \in \partial \Omega_{T} .
\end{array}
$$

Here $\Gamma_{t}$ denotes the (free) interface at the time $t$ with the normal $n$, and the mean curvature $\kappa, \alpha>0$ is the surface tension constant. $D(u)=\frac{1}{2}\left(\nabla u+(\nabla u)^{T}\right)$ denotes the deformation tensor and $I$ is the $d \times d$ identity matrix. [u] denotes the jump of the $u$ across the interface $\Gamma_{t}$. Clearly, (1.8) and (1.9) are the interfacial conditions for the fluid mixture, which are the mathematical descriptions of the balances of the normal stress and the movement. We refer to [10] for the theoretical analysis, in particular, the proof of the local existence theorem, of the sharp interface model (1.6)-(1.11).

Computationally, the above free interface problem is very difficult to approximate due to the existence of the surface tension on the interface. In addition, during the evolution the fluid interface may experience topological changes such as self-intersection, pinch-off, splitting, and fattening. When that happens, the classical solution of the free interface problem ceases to exist; it becomes even more challenging to approximate the problem numerically. 
To overcome the difficulties, an alternative approach for solving interface problems is the diffuse interface (or phase field) theory, which was originally developed as methodology for modeling and approximating solid-liquid phase transitions in which the effects of surface tension and nonequilibrium thermodynamic behavior may be important at the surface [21, 8, 16]. In the theory, the interface is represented as a thin layer of finite thickness. The method uses an auxiliary function (called a phase field function/variable) to indicate the "phase". The phase field function assumes distinct values in the bulk phases away from the interficial regions over which the phase function varies smoothly, and the interface itself can be associated with an intermediate contour or level set of the phase function (cf. 26 and the references therein). It is in this spirit that the diffuse interface model (1.1) -(1.5) and the sharp interface model (1.6)-(1.11) are connected, and it was indeed shown in 24]. (also see 25]) that the former converges to the latter as $\varepsilon \rightarrow 0$.

The paper is organized as follows. In Section 2 we first recall the basic energy law associated with the phase field model (1.1)-(1.5), and then derive some additional a priori energy estimates which show explicit dependence on the physical parameters $\varepsilon, \lambda, \gamma$ and $\nu$. The new a priori energy estimates are necessary for establishing error estimates not only for the proposed numerical methods of this paper but also for any other numerical methods. In Section 3 we propose a continuous in time semi-discrete finite element method for approximating (1.1)-(1.5). The stable $P_{2}-P_{0}$ mixed element is used to discretize the fluid equations, and the continuous quadratic element is employed to discretize the phase equation. It is shown that the proposed semi-discrete finite element method satisfies a discrete energy law which mimics the basic energy law for the differential problem. Optimal order error estimates are also established for the method. Our main idea is to reformulate equation (1.1) by introducing a new "pressure" $\widetilde{p}=p+\frac{\lambda}{2}|\nabla \phi|^{2}+\frac{\lambda}{\varepsilon^{2}} F(\phi)$ in place of the original pressure $p$, and using the $L^{2}$ projection $Q_{h} f\left(\phi_{h}\right)$, instead of $f\left(\phi_{h}\right)$, in the discrete scheme. In Section 4 we propose a fully discrete implicit scheme by discretizing the semi-discrete scheme in time using the backward Euler method. It is proved that the proposed fully discrete implicit scheme also enjoys a (fully) discrete energy law which mimics the basic energy law for the differential problem. It is this (fully) discrete energy law which paves the way for us to establish the convergence of the fully discrete scheme to the phase field model (1.1)-(1.5) as the mesh sizes $h, \tau \rightarrow 0$, and to the sharp interface limiting model (1.6)-(1.11) as the mesh sizes $h, \tau \rightarrow 0$ and the capillary width $\varepsilon \rightarrow 0$. Finally, in Section 5 we present some numerical experiment results to validate our theoretical results and to show the effectiveness of the combined phase field and finite element approach.

\section{A PRIORI ENERGy ESTIMATES}

The standard space notations are used in this paper; we refer to [1, 27] for their exact definitions. In particular, $B^{*}$ denotes the dual space of a Banach space $B$, $(\cdot, \cdot)$ is used to denote the standard $L^{2}(\Omega)$ inner product, $\langle\cdot, \cdot\rangle$ stands for the dual product between $H_{0}^{1}(\Omega)$ and $H^{-1}(\Omega)$, and

$$
\begin{aligned}
& L_{0}^{2}(\Omega)=\left\{q \in L^{2}(\Omega) ;(q, 1)=0\right\}, \quad V=\left\{v \in\left[H_{0}^{1}(\Omega)\right]^{d} ; \operatorname{div} v=0 \text { in } \Omega_{T}\right\}, \\
& H=\left\{v \in\left[L^{2}(\Omega)\right]^{d} ; \operatorname{div} v=0 \text { in } \Omega_{T} \text { and }\left.v \cdot n\right|_{\partial \Omega_{T}}=0\right\}, \quad X=\left[H_{0}^{1}(\Omega)\right]^{d} .
\end{aligned}
$$


In addition, we shall use $c$ to denote a generic positive constant depending only on $\Omega$ and $C$ to denote a generic positive constant depending only on the data $\left(u_{0}^{\varepsilon}, \phi_{0}^{\varepsilon}, g\right.$, $\nu, \lambda, \gamma, T, \Omega)$.

Throughout the paper we will make frequent use of the following known inequalities (cf. [1, 27]):

(2.3) $\|\psi\|_{L^{4}} \leq c\|\psi\|_{L^{2}}^{\frac{4-d}{4}}\|\psi\|_{H^{1}}^{\frac{d}{4}}, \quad \forall \psi \in H^{1}(\Omega)$,

(2.4) $\left\|\nabla^{2} \psi\right\|_{L^{2}} \leq c\|\Delta \psi\|_{L^{2}}, \quad \forall \psi \in H^{2}(\Omega)$ with $\left.\frac{\partial \psi}{\partial n}\right|_{\partial \Omega}=0$,

$(2.5)\|\psi\|_{L^{\infty}} \leq c\|\psi\|_{L^{2}}^{\frac{4-d}{4}}\left(\|\psi\|_{L^{2}}^{2}+\|\Delta \psi\|_{L^{2}}^{2}\right)^{\frac{d}{8}}, \forall \psi \in H^{2}(\Omega)$ with $\left.\frac{\partial \psi}{\partial n}\right|_{\partial \Omega}=0$,

(2.6) $\|\nabla \psi\|_{L^{3}} \leq c\|\nabla \psi\|_{L^{2}}^{\frac{6-d}{6}}\|\Delta \psi\|_{L^{2}}^{\frac{d}{6}}+c\|\nabla \psi\|_{L^{2}}, \forall \psi \in H^{2}(\Omega)$,

$(2.7)\left\|\nabla^{3} \psi\right\|_{L^{2}} \leq c\left(\|\nabla \Delta \psi\|_{L^{2}}+\|\Delta \psi\|_{L^{2}}\right), \quad \forall \psi \in H^{3}(\Omega)$ with $\left.\frac{\partial \psi}{\partial n}\right|_{\partial \Omega}=0$,

where $\tilde{\Delta}=\pi \Delta$ is the Stokes operator and $\pi$ is the $L^{2}$-orthogonal projection from $\left[L^{2}(\Omega)\right]^{d}$ onto $H$.

Existence and uniqueness of weak solutions of system (1.1)-1.5) was proved in 24] (also see [25, 23) using an energy method. A key ingredient of the proof is to establish the basic energy law for the phase field model (see (2.18) below). In this section, after re-establishing the basic energy law, we shall derive some additional a priori estimates for weak solutions to the system (1.1)-(1.5), which will be needed for the error analysis in Section 3 . Special attention will be given to tracing the explicit dependence of the a priori estimates on the capillary width $\varepsilon$.

Lemma 2.1. Suppose that $g \in L^{2}\left((0, T) ;\left[L^{2}(\Omega)\right]^{d}\right)$, and the initial values $u_{0}^{\varepsilon}$ and $\phi_{0}^{\varepsilon}$ satisfy $\left|\phi_{0}^{\varepsilon}\right| \leq 1$ and $\mathcal{E}_{\varepsilon, \lambda}\left(u_{0}^{\varepsilon}, \phi_{0}^{\varepsilon}\right)<\infty$, i.e., the initial energy is bounded. Then every regular solution $(u, \phi, p)$ of system (1.1)-(1.5) satisfies the following estimates: for all $T \in[0, \infty)$ :

$$
\begin{aligned}
& \underset{t \in[0, T]}{\operatorname{ess} \sup }\|\phi(t)\|_{L^{\infty}} \leq 1 \text {, } \\
& \underset{t \in[0, T]}{\operatorname{ess} \sup }\left\{\|u(t)\|_{L^{2}}^{2}+\lambda\|\nabla \phi(t)\|_{L^{2}}^{2}+\lambda \varepsilon^{-2}(F(\phi(t)), 1)\right\} \leq C, \\
& \int_{0}^{T} \nu\|\nabla u(t)\|_{L^{2}}^{2} d t+\left\{\begin{array}{l}
\int_{0}^{T} \lambda \gamma\left\|\Delta \phi(t)-\varepsilon^{-2} f(\phi(t))\right\|_{L^{2}}^{2} d t \\
\int_{0}^{T} \lambda \gamma^{-1}\left\|\phi_{t}(t)+u(t) \cdot \nabla \phi(t)\right\|_{L^{2}}^{2} d t
\end{array}\right\} \leq C, \\
& \int_{0}^{T}\left\|u_{t}(t)\right\|_{\left(V \cap L^{\infty}\right) *}^{2} d t \leq C, \\
& \int_{0}^{T}\left\|\phi_{t}(t)\right\|_{L^{\alpha}}^{\alpha} \leq C \quad \text { for } 1 \leq \alpha<\frac{d}{d-1}
\end{aligned}
$$


where $\widetilde{p}=p+\frac{\lambda}{2}|\nabla \phi|^{2}+\frac{\lambda}{\varepsilon^{2}} F(\phi)$. In addition, there hold

$$
\begin{aligned}
& \int_{0}^{T}\|\Delta \phi(t)\|_{L^{2}}^{2} d t \leq C \varepsilon^{-2}, \\
& \text { ess sup }\left\|\int_{0}^{t} \tilde{p}(s) d s\right\|_{L^{2}} \leq C \varepsilon^{-\frac{d}{6}}, \\
& \int_{0}^{T}\left\|u_{t}(t)\right\|_{V^{*}}^{\frac{12}{6+d}} d t \leq C \varepsilon^{-2} \\
& \int_{0}^{T}\left\|\phi_{t}(t)\right\|_{L^{2}}^{\frac{4}{d}} d t \leq C \varepsilon^{-2} .
\end{aligned}
$$

Proof. First, testing (1.1) with $u$ and (1.2) with $\lambda \gamma^{-1}\left(\phi_{t}+u \cdot \nabla \phi\right)$ or with $\lambda\left(-\Delta \phi+\frac{1}{\varepsilon^{2}} f(\phi)\right)$, using the differential relation

$$
\operatorname{div}(\nabla \phi \otimes \nabla \phi)=\Delta \phi \nabla \phi+\frac{1}{2} \nabla|\nabla \phi|^{2},
$$

and adding the resulting equations lead to the following basic energy law for the phase field model:

$$
\frac{d}{d t} \mathcal{E}_{\varepsilon, \lambda}(u, \phi)=\int_{\Omega} g u d x+\left\{\begin{array}{l}
-\int_{\Omega}\left[\nu|\nabla u|^{2}+\lambda \gamma\left|\Delta \phi-\frac{1}{\varepsilon^{2}} f(\phi)\right|^{2}\right] d x \\
-\int_{\Omega}\left[\nu|\nabla u|^{2}+\lambda \gamma^{-1}\left|\phi_{t}+u \cdot \nabla \phi\right|^{2}\right] d x
\end{array}\right\}
$$

with

$$
\mathcal{E}_{\varepsilon, \lambda}(u, \phi):=\int_{\Omega}\left[\frac{1}{2}|u|^{2}+\frac{\lambda}{2}|\nabla \phi|^{2}+\frac{\lambda}{\varepsilon^{2}} F(\phi)\right] d x .
$$

Next, the assertion (2.8) follows from a weak maximum principle (i.e. testing (1.2) with $\phi^{-}:=\max \{-\phi, 0\}$ and with $\left.(\phi-1)^{+}:=\max \{\phi-1,0\}\right)$, and the estimates (2.9) and (2.10) follow easily from integrating (2.18) in $t$ from 0 to $T$ and using the inequality

$$
|(g, u)| \leq \frac{1}{4}\|u\|_{L^{2}}^{2}+\|g\|_{L^{2}}^{2} .
$$

To show (2.11), using (2.17) we first rewrite equation (1.1) as

$$
u_{t}-\nu \Delta u+\nabla \widetilde{p}+\lambda w \nabla \phi=g, \quad w:=\Delta \phi-\frac{1}{\varepsilon^{2}} f(\phi) .
$$

Then testing the above equation with $v \in V \cap\left[L^{\infty}(\Omega)\right]^{d}$ we have

$$
\begin{aligned}
\left(u_{t}, v\right) & =-\nu(\nabla u, \nabla v)-\lambda(w \nabla \phi, v)+(g, v) \\
& \leq \nu\|\nabla u\|_{L^{2}}\|\nabla v\|_{L^{2}}+\lambda\|w\|_{L^{2}}\|\nabla \phi\|_{L^{2}}\|v\|_{L^{\infty}}+\|g\|_{H^{-1}}\|v\|_{H^{1}} .
\end{aligned}
$$

It follows from (2.21), (2.9), and (2.10) that

$$
\int_{0}^{T}\left\|u_{t}(t)\right\|_{\left(V \cap L^{\infty}\right)^{*}}^{2} d t \leq C
$$

hence (2.11) holds.

Next, noting that (1.2) can be rewritten as

$$
\phi_{t}=\gamma w-u \cdot \nabla \phi,
$$


then (2.12) follows from (2.9), (2.10), and the following inequality:

$$
\|u \cdot \nabla \phi\|_{L^{\alpha}}^{\alpha} \leq\|u\|_{L^{s}}^{\alpha}\|\nabla \phi\|_{L^{\frac{s \alpha}{s-\alpha}}}^{\alpha}
$$

for $1 \leq \alpha<\frac{d}{d-1}, \alpha<s<\frac{2 d}{d-2}$.

To show (2.13), testing equation $w=\Delta \phi-\varepsilon^{-2} f(\phi)$ with $\Delta \phi$ we get

$$
\begin{aligned}
\|\Delta \phi\|_{L^{2}}^{2} & =(w, \Delta \phi)+\frac{1}{\varepsilon^{2}}(f(\phi), \Delta \phi) \leq\|w\|_{L^{2}}\|\Delta \phi\|_{L^{2}}-\frac{1}{\varepsilon^{2}}\left(f^{\prime}(\phi),|\nabla \phi|^{2}\right) \\
& \leq \frac{1}{2}\|\Delta \phi\|_{L^{2}}^{2}+\frac{1}{2}\|w\|_{L^{2}}^{2}+\frac{1}{\varepsilon^{2}}\|\nabla \phi\|_{L^{2}}^{2} .
\end{aligned}
$$

Here we have used the fact that $f^{\prime}(\phi)=3 \phi^{2}-1$. The assertion then follows from the above inequality, (2.9), and (2.10).

To show (2.14), we first deduce from equations (1.1) and (2.17) that

$$
(\tilde{p}, \operatorname{div} v)=\left(u_{t}, v\right)+\nu(\nabla u, \nabla v)+\lambda(w \nabla \phi, v)-(g, v),
$$

for all $v \in\left[H_{0}^{1}(\Omega)\right]^{d}$. Then integrating (2.23) in $t$ yields

$$
\begin{aligned}
\left(\int_{0}^{t} \tilde{p}(s) d s, \operatorname{div} v\right)= & \left(u(t)-u_{0}, v\right)+\nu\left(\int_{0}^{t} \nabla u(s) d s, \nabla v\right) \\
& +\lambda\left(\int_{0}^{t} w(s) \nabla \phi(s) d s, v\right)-\left(\int_{0}^{t} g(s) d s, v\right) .
\end{aligned}
$$

Using (2.1)-(2.3) and the inf-sup inequality [18]:

$$
\|q\|_{L^{2}} \leq c \sup _{v \in X} \frac{(\operatorname{div} v, q)}{\|\nabla v\|_{L^{2}}} \quad \forall q \in L_{0}^{2}(\Omega)
$$

we have

$$
\begin{aligned}
\left\|\int_{0}^{t} \tilde{p}(s) d s\right\|_{L^{2}} & \leq c\|u(t)\|_{L^{2}}+c\left\|u_{0}\right\|_{L^{2}}+c \nu \int_{0}^{t}\|\nabla u\|_{L^{2}} d s \\
& +c \lambda \int_{0}^{t}\|w\|_{L^{2}}\|\nabla \phi\|_{L^{3}} d s+c \int_{0}^{t}\|g\|_{H^{-1}} d s .
\end{aligned}
$$

It then follows from (2.6), (2.13), and (2.26) that

$$
\left\|\int_{0}^{t} \tilde{p}(s) d s\right\|_{L^{2}} \leq C \varepsilon^{-\frac{d}{6}} \quad \forall t \in[0, T] .
$$

Hence, (2.14) holds.

To verify (2.15), testing (2.20) with $v \in V$ we have (compare with (2.21)

$$
\begin{aligned}
\left(u_{t}, v\right) & =-\nu(\nabla u, \nabla v)-\lambda(w \nabla \phi, v)+(g, v) \\
& \leq \nu\|\nabla u\|_{L^{2}}\|\nabla v\|_{L^{2}}+\lambda\|w\|_{L^{2}}\|\nabla \phi\|_{L^{3}}\|v\|_{L^{6}}+\|g\|_{H^{-1}}\|v\|_{H^{1}} .
\end{aligned}
$$

It then follows from (2.28) and the interpolation inequality (cf. [1])

$$
\|\nabla \phi\|_{L^{3}} \leq C\|\nabla \phi\|_{L^{2}}^{\frac{6-d}{6}}\|\Delta \phi\|_{L^{2}}^{\frac{d}{6}}+C\|\nabla \phi\|_{L^{2}}
$$

that

$$
\left\|u_{t}\right\|_{V^{*}} \leq C\left\{\|\nabla u\|_{L^{2}}+\|w\|_{L^{2}}\left(\|\nabla \phi\|_{L^{2}}^{\frac{6-d}{6}}\|\Delta \phi\|_{L^{2}}^{\frac{d}{6}}+\|\nabla \phi\|_{L^{2}}\right)\right\} .
$$

Together with (2.9), (2.10), and (2.13), the above estimate implies that

$$
\int_{0}^{T}\left\|u_{t}(t)\right\|_{V^{*}}^{\frac{12}{6+d}} d t \leq C \varepsilon^{-2}
$$


then (2.15) holds.

Finally, it follows from (2.24) and the interpolation inequality that

$$
\begin{aligned}
\left\|\phi_{t}\right\|_{L^{2}} & \leq \gamma\|w\|_{L^{2}}+\|u \cdot \nabla \phi\|_{L^{2}} \\
& \leq \gamma\|w\|_{L^{2}}+\|u\|_{L^{4}}^{2}+\|\nabla \phi\|_{L^{4}}^{2} \\
& \leq \gamma\|w\|_{L^{2}}+c\|u\|_{L^{2}}^{\frac{4-d}{2}}\|\nabla u\|_{L^{2}}^{\frac{d}{2}}+c\|\nabla \phi\|_{L^{2}}^{2}+c\|\nabla \phi\|_{L^{2}}^{\frac{4-d}{2}}\|\Delta \phi\|_{L^{2}}^{\frac{d}{2}},
\end{aligned}
$$

which, together with (2.9), (2.10), and (2.13) immediately yields (2.16).

The next lemma derives a priori estimates in higher norms for $(u, \phi)$ under stronger assumptions on the initial data $\left(u_{0}^{\varepsilon}, \phi_{0}^{\varepsilon}\right)$.

Lemma 2.2. In addition to the assumptions of Lemma 2.1, let $d=2$, and suppose that $u_{0}^{\varepsilon} \in V, \phi_{0}^{\varepsilon} \in H^{2}(\Omega)$. Then every regular solution $(u, \phi)$ of problems (1.1) -(1.5) satisfies the following estimates:

$$
\begin{aligned}
& \nu\|\nabla u(t)\|_{L^{2}}^{2}+\frac{\lambda^{2}}{\gamma}\|\Delta \phi(t)\|_{L^{2}}^{2} \\
& \quad+\int_{0}^{t}\left[\|\Delta u(s)\|_{L^{2}}^{2}+\lambda^{2}\|\nabla \Delta \phi\|_{L^{2}}^{2}\right] d s \leq C \exp \left(\frac{c_{0}}{\varepsilon^{2}}\right), \\
& \int_{0}^{t}\left[\left\|u_{t}(s)\right\|_{L^{2}}^{2}+\|\nabla p(s)\|_{L^{2}}^{2}\right] d s \leq C \exp \left(\frac{c_{0}}{\varepsilon^{2}}\right),
\end{aligned}
$$

for all $0 \leq t \leq T$. Here $c_{0}=c_{0}\left(u_{0}^{\varepsilon}, \phi_{0}^{\varepsilon}, g, \nu, \lambda, \gamma, T, \Omega\right)$ is some positive constant.

Remark 2.1. The idea of the proof is to test equations (1.1) and (1.2) with $u_{t}-\nu \tilde{\Delta} u$ and $\lambda^{2} \gamma^{-1} \Delta^{2} \phi$, respectively. However, it is easy to see that integration by parts in (1.2) will result in the boundary integral term $\lambda^{2} \gamma^{-1} \int_{\partial \Omega} \frac{\partial \Delta \phi}{\partial n}\left(\phi_{t}-\gamma \Delta \phi\right) d \sigma$, which is not easy to control since it involves higher order derivatives of $\phi$ on the boundary. A common strategy to overcome this kind of difficulty in PDE analysis is: first to use a cut-off function technique to eliminate the boundary integral term and to get the desired interior estimates (i.e., estimates on any compact subdomain $\Omega^{\prime}$ of $\Omega$ ); then to get the desired boundary estimates by a boundary straightening technique (cf. $\S 6.2$ and $\S 8.4$ of [17]). The cut-off function technique would use $\left(\lambda^{2} \gamma^{-1} \Delta^{2} \phi\right) \xi$, instead of $\lambda^{2} \gamma^{-1} \Delta^{2} \phi$, to test (1.2), where $\xi$ is a cut-off function which is smooth with compact support in $\Omega$, that is, $\operatorname{supp} \xi \subset \subset \Omega$. As mentioned above, the very reason for using the cut-off function is to kill the boundary integral term. The trade-off is that one now gets some additional interior integral terms which are usually controllable.

To avoid the technicality of using cut-off functions and because our goal is only to show that a priori estimates of the solutions to (1.1)-(1.3) in higher norms depend on $\frac{1}{\varepsilon}$ exponentially, in the following proof we simply ignore the boundary integral term, which implies that we implicitly assume $\phi$ satisfies $\frac{\partial \Delta \phi}{\partial n}=0$ on $\partial \Omega_{T}$. One may regard estimates (2.29) and (2.30) as interior estimates. A proof for the boundary and global estimates can be carried out using the cut-off function technique and the boundary straightening technique (cf. [17, 22]). It is clear that when the interior estimates grow exponentially in $\frac{1}{\varepsilon}$, so do the global estimates. 
Proof. Testing (1.1) with $u_{t}-\nu \tilde{\Delta} u$ and (1.2) with $\lambda^{2} \gamma^{-1} \Delta^{2} \phi$ and adding the resulting equations, we get

$$
\begin{aligned}
&\left\|u_{t}\right\|_{L^{2}}^{2}+\nu \frac{d}{d t}\|\nabla u\|_{L^{2}}^{2}+\nu^{2}\|\tilde{\Delta} u\|_{L^{2}}^{2}+\frac{\lambda^{2}}{2 \gamma} \frac{d}{d t}\|\Delta \phi\|_{L^{2}}^{2}+\lambda^{2}\|\nabla \Delta \phi\|_{L^{2}}^{2} \\
&=\left(g, u_{t}-\nu \tilde{\Delta} u\right)-\lambda\left(\Delta \phi \nabla \phi, u_{t}-\nu \tilde{\Delta} u\right) \\
&+\frac{\lambda^{2}}{\gamma}(\nabla(u \cdot \nabla \phi), \nabla \Delta \phi)+\frac{\lambda^{2}}{\varepsilon^{2}}\left(f^{\prime}(\phi) \nabla \phi, \nabla \Delta \phi\right) .
\end{aligned}
$$

Using (2.1)-(2.3), each term on the right-hand side of (2.31) can be bounded from above as follows:

$$
\begin{aligned}
& \mid\left(g, u_{t}\right.-\nu \tilde{\Delta} u) \mid \leq \frac{1}{6}\left\|u_{t}\right\|_{L^{2}}^{2}+\frac{\nu^{2}}{6}\|\tilde{\Delta} u\|_{L^{2}}^{2}+3\|g\|_{L^{2}}^{2}, \\
&\left|\lambda\left(\Delta \phi \nabla \phi, u_{t}-\nu \tilde{\Delta} u\right)\right| \\
& \leq \frac{1}{6}\left\|u_{t}\right\|_{L^{2}}^{2}+\frac{\nu^{2}}{6}\|\tilde{\Delta} u\|_{L^{2}}^{2}+3 \lambda^{2}\|\nabla \phi\|_{L^{4}}^{2}\|\Delta \phi\|_{L^{4}}^{2} \\
& \leq \frac{1}{6}\left\|u_{t}\right\|_{L^{2}}^{2}+\frac{\nu^{2}}{6}\|\tilde{\Delta} u\|_{L^{2}}^{2}+\frac{\lambda^{2}}{8}\|\nabla \Delta \phi\|_{L^{2}}^{2} \\
&+c \lambda^{2}\left(\|\nabla \phi\|_{L^{4}}^{2}+\|\nabla \phi\|_{L^{4}}^{\frac{8}{4-d}}\right)\|\Delta \phi\|_{L^{2}}^{2} \\
&\left.\frac{\lambda^{2}}{\gamma} \mid \nabla(u \cdot \nabla \phi), \nabla \Delta \phi\right) \mid \\
& \leq \frac{\lambda^{2}}{16}\|\nabla \Delta \phi\|_{L^{2}}^{2}+\frac{4 \lambda^{2}}{\gamma^{2}}\|\nabla(u \cdot \nabla \phi)\|_{L^{2}}^{2} \\
& \leq \frac{\lambda^{2}}{16}\|\nabla \Delta \phi\|_{L^{2}}^{2}+\frac{4 \lambda^{2}}{\gamma^{2}}\left[\|\nabla u\|_{L^{4}}^{2}\|\nabla \phi\|_{L^{4}}^{2}+\|u\|_{L^{\infty}}^{2}\left\|\nabla^{2} \phi\right\|_{L^{2}}^{2}\right] \\
& \leq \frac{\lambda^{2}}{8}\|\nabla \Delta \phi\|_{L^{2}}^{2}+\frac{\nu^{2}}{6}\|\tilde{\Delta} u\|_{L^{2}}^{2} \\
&+c \nu^{-\frac{4 d}{8-2 d}}\left(\frac{\lambda}{\gamma}\right)^{\frac{8}{4-d}}\left(\|\nabla u\|_{L^{2}}^{2}\|\nabla \phi\|_{L^{4}}^{\frac{8}{4-d}}+\|u\|_{L^{2}}^{2}\|\Delta \phi\|_{L^{2}}^{\frac{8}{4-d}}\right) \\
& \frac{\lambda^{2}}{\varepsilon^{2}}\left|\left(f^{\prime}(\phi) \nabla \phi, \nabla \Delta \phi\right)\right| \leq \frac{\lambda^{2}}{8}\|\nabla \Delta \phi\|_{L^{2}}^{2}+\frac{8 \lambda^{2}}{\varepsilon^{4}}\|\nabla \phi\|_{L^{2}}^{2} .
\end{aligned}
$$

Substituting (2.32)-(2.35) with $d=2$ into (2.31), we get

$$
\begin{aligned}
2 \nu \frac{d}{d t}\|\nabla u\|_{L^{2}}^{2}+ & \frac{\lambda^{2}}{\gamma} \frac{d}{d t}\|\Delta \phi\|_{L^{2}}^{2}+\left\|u_{t}\right\|_{L^{2}}^{2}+\nu^{2}\|\tilde{\Delta} u\|_{L^{2}}^{2}+\lambda^{2}\|\nabla \Delta \phi\|_{L^{2}}^{2} \\
& \leq 6\|g\|_{L^{2}}^{2}+\frac{16 \lambda^{2}}{\varepsilon^{4}}\|\nabla \phi\|_{L^{2}}^{2}+d(t) \frac{\lambda^{2}}{\gamma}\|\Delta \phi\|_{L^{2}}^{2},
\end{aligned}
$$

where

$$
\begin{aligned}
d(t)= & c \lambda^{2}\left(\|\phi\|_{H^{1}}\|\Delta \phi\|_{L^{2}}+\|\phi\|_{H^{1}}^{2}\|\Delta \phi\|_{L^{2}}^{2}\right) \\
& +c \nu^{-2} \lambda^{2} \gamma^{-3}\left(\|\nabla u\|_{L^{2}}^{2}\|\phi\|_{H^{1}}^{2}+\|u\|_{L^{2}}^{2}\|\Delta \phi\|_{L^{2}}^{2}\right) .
\end{aligned}
$$


Finally, testing (1.1) with $2 \nabla p$ and using the identity (2.17) we get

$$
\begin{aligned}
& 2\|\nabla p\|_{L^{2}}^{2}=2(g, \nabla p)-2 \lambda(\Delta \phi \nabla \phi, \nabla p)-\lambda\left(\nabla|\nabla \phi|^{2}, \nabla p\right)+2 \nu(\Delta u, \nabla p) \\
& \quad \leq\|\nabla p\|_{L^{2}}^{2}+4 \lambda^{2}\left(\|\Delta \phi\|_{L^{4}}^{2}+\left\|\nabla^{2} \phi\right\|_{L^{4}}^{2}\right)\|\nabla \phi\|_{L^{4}}^{2}+4\|g\|_{L^{2}}^{2}+4 \nu^{2}\|\Delta u\|_{L^{2}}^{2} \\
& \quad \leq\|\nabla p\|_{L^{2}}^{2}+\frac{\lambda^{2}}{2}\|\nabla \Delta \phi\|_{L^{2}}^{2}+c \lambda^{2}\|\phi\|_{H^{1}}^{4}\|\Delta \phi\|_{L^{2}}^{2}+4\|g\|_{L^{2}}^{2}+c \nu^{2}\|\tilde{\Delta} u\|_{L^{2}}^{2},
\end{aligned}
$$

for $d=2$. Integrating (2.36) and (2.37) from 0 to $t$, respectively, and applying Gronwall's inequality and Lemma 2.1 yield (2.29) and (2.30).

Remark 2.2. The estimates of Lemma 2.2 are proved for the case $d=2$. When $d=3$, since $\frac{2 d}{4-d}=6>2$, the estimates only hold locally in time. We also note that the exponential dependence of the bounds on $\frac{1}{\varepsilon^{2}}$ arises from the polynomial dependence on $\frac{1}{\varepsilon^{2}}$ of $\|\Delta \phi\|_{L^{2}\left(\Omega_{T}\right)}^{2}$.

\section{Continuous in time Semi-Discrete Finite Element approximation}

3.1. Formulation of semi-discrete finite element method. Let $\mathcal{T}_{h}$ be a quasiuniform "triangulation" of the domain $\Omega$ of mesh size $0<h<1$ and $\bar{\Omega}=\bigcup_{K \in \mathcal{T}_{h}} \bar{K}$ $\left(K \in \mathcal{T}_{h}\right.$ are tetrahedrons in the case $\left.d=3\right)$. For a nonnegative integer $r$, let $P_{r}(K)$ denote the space of polynomials of degree less than or equal to $r$ on $K$. We introduce the finite element spaces

$$
\begin{aligned}
& X_{h}=\left\{v_{h} \in\left[C^{0}(\bar{\Omega})\right]^{d} \cap\left[H_{0}^{1}(\Omega)\right]^{d} ;\left.v_{h}\right|_{K} \in\left[P_{2}(K)\right]^{d}\right\}, \\
& V_{h}=\left\{v_{h} \in X_{h} ;\left(\operatorname{div} v_{h}, q_{h}\right)=0, \forall q_{h} \in M_{h}\right\}, \\
& M_{h}=\left\{q_{h} \in L_{0}^{2}(\Omega) ;\left.q_{h}\right|_{K} \in P_{0}(K)\right\}, \quad Y_{h}=\left\{\psi_{h} \in C^{0}(\bar{\Omega}) ;\left.\psi_{h}\right|_{K} \in P_{2}(K)\right\} .
\end{aligned}
$$

It is well known that [7, 18, the $P_{2}-P_{0}$ mixed finite element space $\left(X_{h}, M_{h}\right)$ is a stable pair for the Stokes and Navier-Stokes equations since it satisfies the inf-sup condition

$$
\sup _{v_{h} \in X_{h}} \frac{\left(\operatorname{div} v_{h}, q_{h}\right)}{\left\|\nabla v_{h}\right\|_{L^{2}}} \geq c\left\|q_{h}\right\|_{L^{2}}, \quad \forall q_{h} \in M_{h}
$$

To introduce our semi-discrete finite element method, we need some preparations. Define the $L^{2}$-orthogonal projections $P_{h}:\left[L^{2}(\Omega)\right]^{d} \rightarrow V_{h}, Q_{h}: L^{2}(\Omega) \rightarrow Y_{h}$ and $\rho_{h}$ : $L^{2}(\Omega) \rightarrow M_{h}$ such that

$$
\begin{array}{lr}
\left(v-P_{h} v, v_{h}\right)=0 & \forall v \in\left[L^{2}(\Omega)\right]^{d}, v_{h} \in V_{h}, \\
\left(\psi-Q_{h} \psi, \psi_{h}\right)=0 & \forall \psi \in L^{2}(\Omega), \psi_{h} \in Y_{h}, \\
\left(q-\rho_{h} q, q_{h}\right)=0 & \forall q \in L^{2}(\Omega), q_{h} \in M_{h} .
\end{array}
$$

We also define the discrete Laplacian $\Delta_{h}: Y_{h} \rightarrow Y_{h} \cap L_{0}^{2}(\Omega)$ by

$$
\left(\Delta_{h} \phi_{h}, \psi_{h}\right)=-\left(\nabla \phi_{h}, \nabla \psi_{h}\right) \quad \forall \phi_{h}, \psi_{h} \in Y_{h} .
$$


It is well known that 6, 9, 18, 20, the finite element spaces $X_{h}, M_{h}, Y_{h}$ and the operators $P_{h}, Q_{h}$, and $R_{h}$ satisfy the following approximation properties: for $j=1,2,3$,

$$
\begin{aligned}
& \left\|v-P_{h} v\right\|_{L^{2}}+h\left\|\nabla\left(v-P_{h} v\right)\right\|_{L^{2}} \leq c h^{j}|v|_{H^{j}} \quad \forall v \in V \cap\left[H^{j}(\Omega)\right]^{d}, \\
& \left\|q-\rho_{h} q\right\|_{L^{2}} \leq c h|q|_{H^{1}} \quad \forall q \in H^{1}(\Omega), \\
& \left\|\chi-Q_{h} \chi\right\|_{L^{2}}+h\left\|\nabla\left(\chi-Q_{h} \chi\right)\right\|_{L^{2}} \leq c h^{j}|\chi|_{H^{j}} \quad \forall \chi \in H^{j}(\Omega), \\
& \left\|\nabla \psi_{h}\right\|_{L^{4}} \leq c\left(\left\|\nabla \psi_{h}\right\|_{L^{2}}+\left\|\Delta_{h} \psi_{h}\right\|_{L^{2}}\right)^{\frac{d}{4}}\left\|\nabla \psi_{h}\right\|_{L^{2}}^{\frac{4-d}{4}} \quad \forall \psi_{h} \in Y_{h}, \\
& \left\|\psi_{h}\right\|_{L^{\infty}} \leq c\left\|\psi_{h}\right\|_{L^{2}}^{\frac{4-d}{2}}\left(\left\|\psi_{h}\right\|_{L^{2}}^{2}+\left\|\Delta_{h} \psi_{h}\right\|_{L^{2}}^{2}\right)^{\frac{d}{8}} \quad \forall \psi_{h} \in Y_{h} \text { with }\left.\frac{\partial \psi_{h}}{\partial n}\right|_{\partial \Omega}=0, \\
& \left\|\nabla \psi_{h}\right\|_{L^{3}} \leq c\left\|\nabla \psi_{h}\right\|_{L^{2}}^{\frac{6-d}{6}}\left\|\Delta_{h} \psi_{h}\right\|_{L^{2}}^{\frac{d}{6}}+c\left\|\nabla \psi_{h}\right\|_{L^{2}} \quad \forall \psi_{h} \in Y_{h}, \\
& \left\|\nabla \psi_{h}\right\|_{L^{2}} \leq c h^{-1}\left\|\psi_{h}\right\|_{L^{2}} \quad \forall \psi_{h} \in Y_{h}, \\
& \left\|\nabla v_{h}\right\|_{L^{2}} \leq c h^{-1}\left\|v_{h}\right\|_{L^{2}}, \quad\left\|v_{h}\right\|_{L^{\infty}} \leq c h^{-1}\left\|v_{h}\right\|_{L^{2}} \quad \forall v_{h} \in X_{h} .
\end{aligned}
$$

Remark 3.1. The estimates (3.3), (3.8), and (3.9) are the standard results of the finite element spaces [9, 18. Proofs of (3.2) and (3.4) can be found in the papers by Heywood and Rannacher 20 and Bramble and $\mathrm{Xu}$ 6. (3.5)-(3.7) were proved by Heywood and Rannacher in 20] for finite element functions with zero boundary values. By essentially repeating the proof given in [20] one can show that these interpolation inequalities also hold for general finite element functions as stated above.

Recall that the weak formulation of (1.1) - 1.5) is defined as: find $(u(t), \phi(t), p(t))$ such that

$$
\begin{aligned}
& u \in L^{\infty}\left((0, T) ;\left[L^{2}(\Omega)\right]^{d}\right) \cap L^{2}((0, T) ; X) \cap H^{1}\left((0, T) ;\left(V \cap L^{\infty}(\Omega)\right)^{*}\right), \\
& \phi \in L^{\infty}\left((0, T) ; L^{2}(\Omega)\right) \cap L^{2}\left((0, T) ; H^{2}(\Omega)\right) \cap H^{1}\left((0, T) ; L^{2}(\Omega)\right), \\
& p \in L^{2}\left((0, T) ; L_{0}^{2}(\Omega)\right),
\end{aligned}
$$

and for all $(v, \psi, q) \in\left[H_{0}^{1}(\Omega)\right]^{d} \times H^{2}(\Omega) \times L_{0}^{2}(\Omega)$ there hold

$$
\begin{aligned}
\left\langle u_{t}, v\right\rangle+\nu(\nabla u, \nabla v)-(p, \operatorname{div} v)-\lambda(\nabla \phi \otimes \nabla \phi, \nabla v) & =\langle g, v\rangle, \\
\left\langle\phi_{t}, \psi\right\rangle+b(u, \phi, \psi)+\gamma(\nabla \phi, \nabla \psi)+\frac{\gamma}{\varepsilon^{2}}(f(\phi), \psi) & =0, \\
(\operatorname{div} u, q) & =0,
\end{aligned}
$$

with the initial conditions $u(0)=u_{0}^{\varepsilon}$ and $\phi(0)=\phi_{0}^{\varepsilon}$, where

$$
b(u, \phi, \psi)=(u \cdot \nabla \phi, \psi) .
$$

It was shown that (cf. [24, 25]; also see [23] (3.10)-3.12) has a unique weak solution for $\left.g \in L^{2}\left((0, T) ;\left[L^{2}(\Omega)\right]^{d}\right) 1\right]$ Hence, integrating by parts in the fourth term on the left-hand side of (3.10) and using the identity (2.17), equation (3.10) can be rewritten as

$$
\left\langle u_{t}, v\right\rangle+\nu(\nabla u, \nabla v)-(\widehat{p}, \operatorname{div} v)+\lambda b(v, \phi, \Delta \phi)=(g, v),
$$

where

$$
\widehat{p}:=p+\frac{\lambda}{2}|\nabla \phi|^{2}
$$

\footnotetext{
${ }^{1}$ The uniqueness holds for all $\nu>0$ when $d=2$, but it is only proved for sufficiently large $\nu$ when $d=3$.
} 
One may now construct finite element approximations based on the formulation (3.14), (3.11), and (3.12). Unfortunately, such a finite element method does not satisfy a discrete energy law which mimics the basic energy law (2.18) due to the fact that $f\left(\phi_{h}\right)$ is not a finite element function in $Y_{h}$ even though $\phi_{h}$ is. To overcome the difficulty, our idea is to rewrite (3.10) into another equivalent form

$$
\left\langle u_{t}, v\right\rangle+\nu(\nabla u, \nabla v)-(\widetilde{p}, \operatorname{div} v)-\frac{\lambda}{\varepsilon^{2}}(f(\phi) \nabla \phi, v)+\lambda b(v, \phi, \Delta \phi)=(g, v)
$$

where

$$
\widetilde{p}:=p+\frac{\lambda}{2}|\nabla \phi|^{2}+\frac{\lambda}{\varepsilon^{2}} F(\phi) .
$$

It is interesting to note that the sum of the last two terms in the definition of $\tilde{p}$ is exactly the energy density function for the Allen-Cahn equation (cf. [12]). We also remark that the idea of introducing the "new pressure" $\widetilde{p}$ is also used in 3 ] to develop a convergent fully discrete finite element method for an Ericksen-Leslie model for the flow of liquid crystals.

Based on the new weak formulation (3.16), (3.11), and (3.12), our semi-discrete finite element approximation of problems (1.1)-(1.5) is defined as follows: find $\left(u_{h}(t), \phi_{h}(t), \tilde{p}_{h}(t)\right) \in X_{h} \times Y_{h} \times M_{h}$ such that for all $t \in(0, T]$ and $\left(v_{h}, \psi_{h}, q_{h}\right) \in$ $X_{h} \times Y_{h} \times M_{h}$

$$
\begin{aligned}
& \left(u_{h t}, v_{h}\right)+\nu\left(\nabla u_{h}, \nabla v_{h}\right)-\left(\widetilde{p}_{h}, \operatorname{div} v_{h}\right) \\
& \quad-\frac{\lambda}{\varepsilon^{2}}\left(Q_{h} f\left(\phi_{h}\right) \nabla \phi_{h}, v_{h}\right)+\lambda b\left(v_{h}, \phi_{h}, \Delta_{h} \phi_{h}\right)=\left(g, v_{h}\right), \\
& \left(\phi_{h t}, \psi_{h}\right)+b\left(u_{h}, \phi_{h}, \psi_{h}\right)+\gamma\left(\nabla \phi_{h}, \nabla \psi_{h}\right)+\frac{\gamma}{\varepsilon^{2}}\left(f\left(\phi_{h}\right), \psi_{h}\right)=0, \\
& \left(\operatorname{div} u_{h}, q_{h}\right)=0,
\end{aligned}
$$

with the initial conditions $u_{h}(0)=u_{0 h}:=P_{h} u_{0}^{\varepsilon}$ and $\phi_{h}(0)=\phi_{0 h}:=Q_{h} \phi_{0}^{\varepsilon}$.

The most important property of the semi-discrete scheme (3.18)-(3.20) is the following discrete energy law.

Lemma 3.1. Let $\left(u_{h}, \phi_{h}, \widetilde{p}_{h}\right)$ solve (3.18)-3.20). Then it holds that

$$
\begin{aligned}
\mathcal{E}_{\varepsilon, \lambda}\left(u_{h}(t), \phi_{h}(t)\right)+\int_{0}^{t}\left\{\nu\left\|\nabla u_{h}(s)\right\|_{L^{2}}^{2}+\lambda \gamma \| Q_{h}\left[\Delta_{h} \phi_{h}(s)\right.\right. \\
\left.\left.\quad-\varepsilon^{-2} f\left(\phi_{h}(s)\right)\right] \| L^{2^{2}}\right\} d s=\mathcal{E}_{\varepsilon, \lambda}\left(u_{h}(0), \phi_{h}(0)\right)+\int_{0}^{t}\left(g(s), u_{h}(s)\right) d s
\end{aligned}
$$

for all $t \in[0, T]$. Here $\mathcal{E}_{\varepsilon, \lambda}(\cdot, \cdot)$ is defined by (2.19).

Proof. Note that if we let

$$
w_{h}:=Q_{h}\left[\Delta_{h} \phi_{h}-\varepsilon^{-2} f\left(\phi_{h}\right)\right]
$$

then (3.18)-(3.19) can be rewritten as

$$
\begin{aligned}
\left(u_{h t}, v_{h}\right)+\nu\left(\nabla u_{h}, \nabla v_{h}\right)-\left(\widetilde{p}_{h}, \operatorname{div} v_{h}\right)+\lambda b\left(v_{h}, \phi_{h}, w_{h}\right) & =\left(g, v_{h}\right), \\
\left(\phi_{h t}, \psi_{h}\right)+b\left(u_{h}, \phi_{h}, \psi_{h}\right)-\gamma\left(w_{h}, \psi_{h}\right) & =0 .
\end{aligned}
$$

The desired identity (3.21) can be obtained by setting $v_{h}=u_{h}$ in (3.23), $\psi_{h}=$ $-\lambda w_{h}$ in (3.24),$q_{h}=\widetilde{p}_{h}$ in (3.20), testing (3.22) with $\frac{\partial \phi_{h}}{\partial t}$, adding the resulting equations, and integrating the sum from 0 to $t$. 
Remark 3.2. (a) The discrete energy law (3.21) and an application of the fixed point theorem in finite-dimensional spaces immediately imply the solvability of the scheme (3.18)-(3.20).

(b) The above proof actually suggests how to implement the scheme (3.18)(3.20) in practice. That is, $w_{h}$ should be formed in each time step (cf. Section 4) by solving the following equation:

$$
\left(w_{h}, \chi_{h}\right)=-\left(\nabla \phi_{h}, \nabla \chi_{h}\right)-\frac{1}{\varepsilon^{2}}\left(f\left(\phi_{h}\right), \chi_{h}\right) \quad \forall \chi_{h} \in Y_{h} .
$$

Lemma 3.2. Suppose that $g \in L^{2}\left((0, T) ;\left[L^{2}(\Omega)\right]^{d}\right)$, and the initial values $u_{0}^{\varepsilon}$ and $\phi_{0}^{\varepsilon}$ satisfy $\left|\phi_{0}^{\varepsilon}\right| \leq 1$ and $\mathcal{E}_{\varepsilon, \lambda}\left(u_{0}^{\varepsilon}, \phi_{0}^{\varepsilon}\right)<\infty$, and let $\left(u_{h}, \phi_{h}, \widetilde{p}_{h}\right)$ solve (3.18)-(3.20). Then there hold

$$
\begin{aligned}
& \underset{t \in[0, T]}{\operatorname{ess} \sup }\left\{\left\|u_{h}(t)\right\|_{L^{2}}^{2}+\lambda\left\|\nabla \phi_{h}(t)\right\|_{L^{2}}^{2}+\lambda \varepsilon^{-2}\left(F\left(\phi_{h}(t)\right), 1\right)\right\} \leq C, \\
& \int_{0}^{T} \nu\left\|\nabla u_{h}(t)\right\|_{L^{2}}^{2} d t+\lambda \gamma \int_{0}^{T}\left\|Q_{h}\left[\Delta_{h} \phi_{h}(t)-\varepsilon^{-2} f\left(\phi_{h}(t)\right)\right]\right\|_{L^{2}}^{2} d t \leq C, \\
& \int_{0}^{T}\left\|u_{h t}\right\|_{\left(V \cap L^{\infty}\right)^{*}}^{2} d t \leq C, \\
& \int_{0}^{T}\left\|\phi_{h t}(t)\right\|_{L^{\alpha}}^{\alpha} \leq C \quad \text { for } 1 \leq \alpha<\frac{d}{d-1}, \\
& \lambda \gamma \int_{0}^{t}\left\|\Delta_{h} \phi_{h}\right\|_{L^{2}}^{2} d s \leq C \varepsilon^{-4}, \\
& \operatorname{ess} \sup \left\|\int_{t \in[0, T]}^{t} \tilde{p}_{h}(s) d s\right\|_{L^{2}} \leq C \varepsilon^{-\frac{d}{3}} .
\end{aligned}
$$

Proof. (3.25) and (3.26) follow immediately from an application of Young's inequality and (3.21).

To show (3.27), for any $v \in V \cap\left[L^{\infty}(\Omega)\right]^{d}$ setting $v_{h}=P_{h} v$ in (3.23) and using (3.2) we get

$$
\begin{gathered}
\left(u_{h t}, v\right)=-\nu\left(\nabla u_{h}, \nabla P_{h} v\right)-\lambda b\left(P_{h} v-v+v, \phi_{h}, w_{h}\right)+\left(g, P_{h} v\right) \\
\leq c \nu\left\|\nabla u_{h}\right\|_{L^{2}}\|\nabla v\|_{L^{2}}+\lambda\left\|w_{h}\right\|_{L^{2}}\left\|\nabla \phi_{h}\right\|_{L^{2}}\|v\|_{L^{\infty}} \\
\quad+\lambda\left\|w_{h}\right\|_{L^{\infty}}\left\|\nabla \phi_{h}\right\|_{L^{2}}\left\|P_{h} v-v\right\|_{L^{2}}+c\|g\|_{H^{-1}}\|\nabla v\|_{L^{2}} \\
\leq c \nu\left\|\nabla u_{h}\right\|_{L^{2}}\|\nabla v\|_{L^{2}}+c \lambda\left\|w_{h}\right\|_{L^{2}}\left\|\nabla \phi_{h}\right\|_{L^{2}}\left(\|v\|_{L^{\infty}}+\|\nabla v\|_{L^{2}}\right) \\
\quad+c\|g\|_{H^{-1}}\|\nabla v\|_{L^{2}} .
\end{gathered}
$$

It follows from the above estimate and (3.25)-(3.26) that

$$
\int_{0}^{T}\left\|u_{h t}\right\|_{\left(V \cap L^{\infty}\right) *}^{2} \leq C,
$$

hence, (3.27) holds.

To verify (3.28), let $\alpha^{\prime}$ denote the conjugate of $\alpha$, for any $\psi \in L^{\alpha^{\prime}}(\Omega)$ setting $\psi_{h}=Q_{h} \psi$ in (3.24), and using (3.5) we get

$$
\begin{aligned}
\left(\phi_{h t}, \psi\right) & =\gamma\left(w_{h}, \psi\right)-b\left(u_{h}, \phi_{h}, Q_{h} \psi\right) \\
\leq & \gamma\left\|w_{h}\right\|_{L^{\alpha}}\|\psi\|_{L^{\alpha^{\prime}}}+\left\|u_{h} \nabla \phi_{h}\right\|_{L^{\alpha}}\|\psi\|_{L^{\alpha^{\prime}}} .
\end{aligned}
$$

Hence,

$$
\left\|\phi_{h t}\right\|_{L^{\alpha}} \leq \gamma\left\|w_{h}\right\|_{L^{\alpha}}+\left\|u_{h} \nabla \phi_{h}\right\|_{L^{\alpha}}
$$


which along with (3.25)-(3.26) imply (3.28).

To show (3.29) and (3.30), we deduce from (3.6), (3.22), and (3.25) that

$$
\begin{aligned}
\left\|\phi_{h}\right\|_{L^{2}}^{2} & =\int_{\Omega}\left(\phi_{h}^{2}-1\right) d x+|\Omega| \leq C \\
\left\|\Delta_{h} \phi_{h}\right\|_{L^{2}}^{2} & =\left(w_{h}, \Delta_{h} \phi_{h}\right)+\varepsilon^{-2}\left(f\left(\phi_{h}\right), \Delta_{h} \phi_{h}\right) \\
& \leq \frac{1}{2}\left\|\Delta_{h} \phi_{h}\right\|_{L^{2}}^{2}+\left\|w_{h}\right\|_{L^{2}}^{2}+\varepsilon^{-4}\left\|\left(\phi_{h}^{2}-1\right) \phi_{h}\right\|_{L^{2}}^{2} \\
& \leq \frac{1}{2}\left\|\Delta_{h} \phi_{h}\right\|_{L^{2}}^{2}+\left\|w_{h}\right\|_{L^{2}}^{2}+C \varepsilon^{-4}\left\{\left\|\nabla \phi_{h}\right\|_{L^{2}}^{6}+\left\|\phi_{h}\right\|_{L^{2}}^{2}\right\} \\
& \leq \frac{1}{2}\left\|\Delta_{h} \phi_{h}\right\|_{L^{2}}^{2}+\left\|w_{h}\right\|_{L^{2}}^{2}+C \varepsilon^{-4} .
\end{aligned}
$$

Integrating (3.32) from 0 to $t$ and using (3.26) we have

$$
\lambda \gamma \int_{0}^{t}\left\|\Delta_{h} \phi_{h}\right\|_{L^{2}}^{2} d s \leq C \varepsilon^{-4}
$$

hence, (3.29) holds.

Integrating (3.23) from 0 to $t$ we find

$$
\begin{aligned}
\left(\int_{0}^{t} \widetilde{p}_{h} d s, \operatorname{div} v_{h}\right)=( & \left.u_{h}(t)-u_{0 h}, v_{h}\right)+\nu\left(\int_{0}^{t} \nabla u_{h} d s, \nabla v_{h}\right) \\
& +\lambda\left(\int_{0}^{t} w_{h} \nabla \phi_{h} d s, v_{h}\right)-\left(\int_{0}^{t} g d s, v_{h}\right) .
\end{aligned}
$$

It follows from (2.1), (3.1), (3.7), (3.25) $-(3.26)$, and (3.33)-(3.34) that

$$
\begin{aligned}
& \left\|\int_{0}^{t} \tilde{p}_{h} d s\right\|_{L^{2}} \leq c\left\|u_{h}(t)\right\|_{L^{2}}+c\left\|u_{0}\right\|_{L^{2}}+c \nu T^{\frac{1}{2}}\left(\int_{0}^{t}\left\|\nabla u_{h}\right\|_{L^{2}}^{2} d s\right)^{\frac{1}{2}} \\
& \quad+c \lambda\left(\int_{0}^{t}\left\|w_{h}\right\|_{L^{2}}^{2} d s\right)^{\frac{1}{2}}\left(\int_{0}^{t}\left\|\nabla \phi_{h}\right\|_{L^{3}}^{2} d s\right)^{\frac{1}{2}}+c T^{\frac{1}{2}}\left(\int_{0}^{t}\|g\|_{L^{2}}^{2} d s\right)^{\frac{1}{2}} \\
& \leq C+c \lambda\left(\int_{0}^{t}\left\|w_{h}\right\|_{L^{2}}^{2} d s\right)^{\frac{1}{2}}\left(\int_{0}^{t}\left\|\nabla \phi_{h}\right\|_{L^{2}}^{2} d s\right)^{\frac{1}{2}} \\
& \quad+c \lambda\left(\int_{0}^{t}\left\|w_{h}\right\|_{L^{2}}^{2} d s\right)^{\frac{1}{2}}\left(\int_{0}^{t}\left\|\nabla \phi_{h}\right\|_{L^{2}}^{2} d s\right)^{\frac{6-d}{12}}\left(\int_{0}^{t}\left\|\Delta_{h} \phi_{h}\right\|_{L^{2}}^{2} d s\right)^{\frac{d}{12}} \\
& \leq C \varepsilon^{-\frac{d}{3}} \quad \forall t \in[0, T],
\end{aligned}
$$

which gives (3.30). The proof is complete.

3.2. Error estimates for the semi-discrete finite element method. The goal of this subsection is to establish optimal order error estimates for the solution $\left(u_{h}, \phi_{h}, p_{h}\right)$ of the semi-discrete scheme (3.18)-(3.20). The analysis involves some delicate applications of norm interpolation results in Sobolev spaces in order to handle the nonlinear coupling terms in the error equations. 
Theorem 3.1. Under the assumptions of Lemma 2.2, it holds that

$$
\begin{aligned}
& \int_{0}^{T}\left(\nu\left\|\nabla\left(u(t)-u_{h}(t)\right)\right\|_{L^{2}}^{2}+\lambda \gamma\left\|\Delta \phi(t)-\Delta_{h} \phi_{h}(t)\right\|_{L^{2}}^{2}\right) d t \\
& \quad+\sup _{0 \leq t \leq T}\left\{\left\|u(t)-u_{h}(t)\right\|_{L^{2}}^{2}+\lambda\left\|\phi(t)-\phi_{h}(t)\right\|_{H^{1}}^{2}\right\} \leq C \varepsilon^{-4} e^{c_{0} \varepsilon^{-3}} h^{2}, \\
& \left\|\int_{0}^{t}\left(\widetilde{p}(t)-\widetilde{p}_{h}(t)\right) d t\right\|_{L^{2}}^{2} \leq C \varepsilon^{-4} e^{c_{0} \varepsilon^{-3}} h^{2} .
\end{aligned}
$$

Proof. Subtracting (3.18) from (3.16) with $v=v_{h}$, (3.19) from (3.11) with $\phi=\psi_{h}$, and (3.20) from (3.12) with $q=q_{h}$, we obtain the following error equations:

$$
\begin{aligned}
\left(u_{t}-u_{h t}, v_{h}\right) & +\nu\left(\nabla\left(u-u_{h}\right), \nabla v_{h}\right)-\left(\widetilde{p}-\widetilde{p}_{h}, \operatorname{div} v_{h}\right)+\left(q_{h}, \operatorname{div}\left(u-u_{h}\right)\right) \\
+ & \lambda b\left(v_{h}, \phi-\phi_{h}, \Delta \phi\right)+\lambda b\left(v_{h}, \phi_{h}, \Delta \phi-\Delta_{h} \phi_{h}\right) \\
& -\frac{\lambda}{\varepsilon^{2}}\left(\left(f(\phi)-Q_{h} f\left(\phi_{h}\right)\right) \nabla \phi+Q_{h} f\left(\phi_{h}\right) \nabla\left(\phi-\phi_{h}\right), v_{h}\right)=0, \\
\left(\phi_{t}-\phi_{h t}, \psi_{h}\right)- & \gamma\left(\Delta \phi-\Delta_{h} \phi_{h}, \psi_{h}\right)+b\left(u-u_{h}, \phi, \psi_{h}\right)+b\left(u_{h}, \phi-\phi_{h}, \psi_{h}\right) \\
& +\frac{\gamma}{\varepsilon^{2}}\left(f(\phi)-f\left(\phi_{h}\right), \psi_{h}\right)=0,
\end{aligned}
$$

for all $\left(v_{h}, \psi_{h}, q_{h}\right) \in\left(X_{h}, Y_{h}, M_{h}\right)$. Setting $\left(e_{u}, e_{\phi}, e_{p}\right)=\left(P_{h} u-u_{h}, Q_{h} \phi-\phi_{h}, \rho_{h} \widetilde{p}-\right.$ $\left.\widetilde{p}_{h}\right)$ and taking $\left(v_{h}, q_{h}\right)=\left(e_{u}, e_{p}\right)$ in (3.37), $\psi_{h}=\lambda\left(e_{\phi}-\Delta_{h} e_{\phi}\right)$ in (3.38), and adding these two relations, we obtain

$$
\begin{aligned}
\frac{1}{2} \frac{d}{d t}\{\| & \left.e_{u}\left\|_{L^{2}}^{2}+\lambda\right\| e_{\phi} \|_{H^{1}}^{2}\right)+\nu\left\|\nabla e_{u}\right\|_{L^{2}}^{2}+\lambda \gamma\left(\left\|\nabla e_{\phi}\right\|_{L^{2}}^{2}+\left\|\Delta_{h} e_{\phi}\right\|_{L^{2}}^{2}\right\} \\
& +\lambda b\left(e_{u}, e_{\phi}, \Delta \phi\right)+\lambda b\left(e_{u}, \phi_{h}, \Delta_{h} e_{\phi}\right)+\lambda b\left(e_{u}, \phi, e_{\phi}-\Delta_{h} e_{\phi}\right) \\
& +\lambda b\left(u_{h}, e_{\phi}, e_{\phi}-\Delta_{h} e_{\phi}\right)+\frac{\lambda \gamma}{\varepsilon^{2}}\left(f(\phi)-f\left(\phi_{h}\right), e_{\phi}-\Delta_{h} e_{\phi}\right) \\
& -\frac{\lambda}{\varepsilon^{2}}\left(f(\phi) \nabla\left(\phi-\phi_{h}\right)+\left(f(\phi)-f\left(\phi_{h}\right)\right) \nabla \phi_{h}+\left(f\left(\phi_{h}\right)-Q_{h} f\left(\phi_{h}\right) \nabla \phi_{h}, e_{u}\right)\right. \\
= & \lambda b\left(e_{u}, Q_{h} \phi-\phi, \Delta \phi\right)+\lambda b\left(e_{u}, \phi_{h}, \Delta_{h} Q_{h} \phi-\Delta \phi\right) \\
& +\lambda b\left(P_{h} u-u, \phi, e_{\phi}-\Delta_{h} e_{\phi}\right) \\
& +\lambda b\left(u_{h}, Q_{h} \phi-\phi, e_{\phi}-\Delta_{h} e_{\phi}\right) \\
& +\left(\widetilde{p}-\rho_{h} \widetilde{p}, \operatorname{div} e_{u}\right) \\
& +\nu\left(\nabla e_{u}, \nabla\left(P_{h} u-u\right)\right) \\
& +\lambda \gamma\left(\nabla e_{\phi}, \nabla\left(Q_{h} \phi-\phi\right)\right)+\lambda \gamma\left(\Delta_{h} e_{\phi}, \Delta_{h} Q_{h} \phi-\Delta \phi\right) .
\end{aligned}
$$

By (2.1), (3.5)-(3.9) and some direct calculations we get

$$
\begin{aligned}
& \frac{\lambda \gamma}{\varepsilon^{2}}\left(f(\phi)-f\left(\phi_{h}\right), e_{\phi}\right) \\
& =\frac{\lambda \gamma}{\varepsilon^{2}}\left[\left\|\phi-\phi_{h}\right\|_{L^{4}}^{4}+3\left\|\phi\left(\phi-\phi_{h}\right)\right\|_{L^{2}}^{2}-3\left(\phi\left(\phi-\phi_{h}\right),\left(\phi-\phi_{h}\right)^{2}\right)-\left\|e_{\phi}\right\|_{L^{2}}^{2}\right] \\
& \quad \quad-\frac{\lambda \gamma}{\varepsilon^{2}}\left(\left(\phi-\phi_{h}\right)^{3}+3 \phi^{2}\left(\phi-\phi_{h}\right)-3 \phi\left(\phi-\phi_{h}\right)^{2}, \phi-Q_{h} \phi\right) \\
& \geq-c \frac{\lambda \gamma}{\varepsilon^{2}}\left(1+\left\|\phi-Q_{h} \phi\right\|_{H^{1}}^{2}\right)\left\|\phi-Q_{h} \phi\right\|_{L^{2}}^{2}-\frac{\lambda \gamma}{\varepsilon^{2}}\left\|e_{\phi}\right\|_{L^{2}}^{2},
\end{aligned}
$$




$$
\begin{aligned}
& \frac{\lambda \gamma}{\varepsilon^{2}}\left(f(\phi)-f\left(\phi_{h}\right),-\Delta_{h} e_{\phi}\right) \\
& \geq \frac{\lambda \gamma}{\varepsilon^{2}}\left(2 \phi^{2}-2-\phi\left(\phi-\phi_{h}\right)+\phi_{h}^{2}-1,-\Delta_{h} e_{\phi}\left(\phi-\phi_{h}\right)\right)+2 \frac{\lambda \gamma}{\varepsilon^{2}}\left\|\nabla e_{\phi}\right\|_{L^{2}}^{2} \\
& \geq 2 \frac{\lambda \gamma}{\varepsilon^{2}}\left\|\nabla e_{\phi}\right\|_{L^{2}}^{2}-\frac{\lambda \gamma}{\varepsilon^{2}}\left\|2 \phi^{2}-2+\phi_{h}^{2}-1\right\|_{L^{4}}\left\|\phi-\phi_{h}\right\|_{L^{4}}\left\|\Delta_{h} e_{\phi}\right\|_{L^{2}} \\
& -\frac{\lambda \gamma}{\varepsilon^{2}}\left\|\phi-\phi_{h}\right\|_{L^{4}}^{2}\left\|\Delta_{h} e_{\phi}\right\|_{L^{2}} \\
& \geq 2 \frac{\lambda \gamma}{\varepsilon^{2}}\left\|\nabla e_{\phi}\right\|_{L^{2}}^{2}-\frac{\lambda \gamma}{8}\left\|\Delta_{h} e_{\phi}\right\|_{L^{2}}^{2} \\
& -\frac{c \gamma}{\varepsilon^{2}}\left(\varepsilon^{-2}\left\|2 \phi^{2}-2+\phi_{h}^{2}-1\right\|_{L^{4}}^{2}+\left\|\Delta_{h} e_{\phi}\right\|_{L^{2}}\right) \lambda\left\|\phi-\phi_{h}\right\|_{H^{1}}^{2}, \\
& \frac{\lambda}{\varepsilon^{2}}\left|\left(f(\phi) \nabla\left(\phi-\phi_{h}\right), e_{u}\right)\right| \leq \frac{\lambda \gamma}{\varepsilon^{2}}\left(\left\|\nabla e_{\phi}\right\|_{L^{2}}^{2}+\left\|\nabla\left(\phi-Q_{h} \phi\right)\right\|_{L^{2}}^{2}\right)+\frac{c \lambda}{\gamma \varepsilon^{2}}\left\|e_{u}\right\|_{L^{2}}^{2}, \\
& \frac{\lambda}{\varepsilon^{2}}\left|\left(\left(f(\phi)-f\left(\phi_{h}\right)\right) \nabla \phi_{h}, e_{u}\right)\right| \leq \frac{c \lambda}{\varepsilon^{2}}\left\|\nabla \phi_{h}\right\|_{L^{4}}\left\|\phi-\phi_{h}\right\|_{L^{4}}\left\|e_{u}\right\|_{L^{2}} \\
& +\frac{c \lambda}{\varepsilon^{2}}\left\|\nabla \phi_{h}\right\|_{L^{4}}\left(\left\|\phi-\phi_{h}\right\|_{L^{4}}+\left\|\phi_{h}^{2}-1\right\|_{L^{4}}\right)\left\|\phi-\phi_{h}\right\|_{L^{4}}\left\|e_{u}\right\|_{L^{4}} \\
& \leq \frac{\nu}{8}\left\|\nabla e_{u}\right\|_{L^{2}}^{2}+\frac{c \lambda^{2}}{\nu \varepsilon^{4}}\left\|\phi_{h}^{2}-1\right\|_{L^{2}}^{2}\left\|\nabla \phi_{h}\right\|_{L^{4}}^{2}\left\|\phi-\phi_{h}\right\|_{H^{1}}^{2} \\
& +\frac{c}{\varepsilon^{2}}\left(\left\|\nabla e_{u}\right\|_{L^{2}}^{2}+\left\|\phi_{h}^{2}-1\right\|_{H^{1}}^{2}+\left\|\nabla \phi_{h}\right\|_{L^{4}}^{2}\right)\left(\lambda\left\|\phi-\phi_{h}\right\|_{H^{1}}^{2}+\lambda\left\|e_{u}\right\|_{L^{2}}^{2}\right), \\
& \frac{\lambda}{\varepsilon^{2}}\left|\left(\left(f\left(\phi_{h}\right)-Q_{h} f\left(\phi_{h}\right)\right) \nabla \phi_{h}, e_{u}\right)\right| \leq c \frac{\lambda}{\varepsilon^{2}} h\left\|\nabla f\left(\phi_{h}\right)\right\|_{L^{2}}\left\|\nabla \phi_{h}\right\|_{L^{4}}\left\|e_{u}\right\|_{L^{4}} \\
& \leq \frac{\nu}{8}\left\|\nabla e_{u}\right\|^{2}+\nu\left(\left\|\nabla \phi_{h}\right\|_{L^{2}}^{2}+\left\|\Delta_{h} \phi_{h}\right\|_{L^{2}}^{2}\right)\left\|e_{u}\right\|_{L^{2}}^{2} \\
& +\frac{c \lambda^{2} h^{2}}{\nu \varepsilon^{4}}\left(\left\|\left(\phi_{h}^{2}-1\right)\right\|_{L^{4}}^{2}\left\|\phi_{h}\right\|_{L^{4}}^{2}+\left\|\nabla \phi_{h}\right\|_{L^{2}}^{2}\right)\left(1+\left\|\nabla \phi_{h}\right\|_{L^{2}}^{2}\right), \\
& \left\|\left(\phi^{2}-1\right)\right\|_{H^{1}}^{2} \leq\left\|\phi^{2}-1\right\|_{L^{2}}^{2}+4\|\phi \nabla \phi\|_{L^{2}}^{2}, \\
& \left\|\left(\phi^{2}-1\right)\right\|_{L^{4}}^{2} \leq\left\|\left(\phi^{2}-1\right)\right\|_{L^{2}}\left\|\left(\phi^{2}-1\right)\right\|_{H^{1}} \\
& \left\|\left(\phi_{h}^{2}-1\right)\right\|_{L^{4}}^{2} \leq\left\|\left(\phi_{h}^{2}-1\right)\right\|_{L^{2}}\left\|\left(\phi_{h}^{2}-1\right)\right\|_{H^{1}}, \\
& \left\|\nabla \phi_{h}\right\|_{L^{4}}^{2} \leq c\left\|\nabla \phi_{h}\right\|_{L^{2}}\left\|\Delta_{h} \phi_{h}\right\|_{L^{2}}+c\left\|\nabla \phi_{h}\right\|_{L^{2}}^{2}, \\
& \left\|\Delta_{h} Q_{h} \phi\right\|_{L^{2}}=\sup _{\psi_{h} \in Y_{h}} \frac{\left(\Delta_{h} Q_{h} \phi-\Delta \phi, \psi_{h}\right)+\left(\Delta \phi, \psi_{h}\right)}{\left\|\psi_{h}\right\|_{L^{2}}} \\
& \leq c\left(h^{-1}\left\|\nabla\left(Q_{h} \phi-\phi\right)\right\|_{L^{2}}+\|\Delta \phi\|_{L^{2}}\right) \leq c\|\Delta \phi\|_{L^{2}}, \\
& \left\|\Delta \phi-\Delta_{h} Q_{h} \phi\right\|_{L^{2}} \leq\left\|\Delta \phi-Q_{h} \Delta \phi\right\|_{L^{2}}+\sup _{\psi_{h} \in Y_{h}} \frac{\left(\Delta \phi-\Delta_{h} Q_{h} \phi, \psi_{h}\right)}{\left\|\psi_{h}\right\|_{L^{2}}}
\end{aligned}
$$

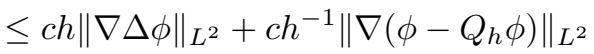

$$
\begin{aligned}
& \leq \operatorname{ch}\left(\|\Delta \phi\|_{L^{2}}+\|\nabla \Delta \phi\|_{L^{2}}\right), \\
& \lambda\left|b\left(e_{u}, e_{\phi}, \Delta \phi\right)\right| \leq c \lambda\left\|e_{u}\right\|_{L^{4}}\left\|\nabla e_{\phi}\right\|_{L^{4}}\|\Delta \phi\|_{L^{2}} \\
& \leq \frac{1}{16}\left(\nu\left\|\nabla e_{u}\right\|_{L^{2}}^{2}+\lambda \gamma\left\|\Delta_{h} e_{\phi}\right\|_{L^{2}}^{2}\right) \\
& +c \lambda\left(\nu^{-1}+\gamma^{-1}\right)\|\Delta \phi\|_{L^{2}}^{2}\left(\lambda\left\|e_{\phi}\right\|_{H^{1}}^{2}+\left\|e_{u}\right\|_{L^{2}}^{2}\right),
\end{aligned}
$$




$$
\begin{aligned}
\lambda\left|b\left(e_{u}, \phi_{h}, \Delta_{h} e_{\phi}\right)\right| \leq & c \lambda\left\|e_{u}\right\|_{L^{4}}\left\|\nabla \phi_{h}\right\|_{L^{4}}\left\|\Delta_{h} e_{\phi}\right\|_{L^{2}} \\
\leq & \frac{1}{16}\left(\nu\left\|\nabla e_{u}\right\|_{L^{2}}^{2}+\lambda \gamma\left\|\Delta_{h} e_{\phi}\right\|_{L^{2}}^{2}\right) \\
& +\frac{c \lambda^{2}}{\nu \gamma^{2}}\left\|\nabla \phi_{h}\right\|_{L^{2}}^{2}\left(\left\|\nabla \phi_{h}\right\|^{2}+\left\|\Delta_{h} \phi_{h}\right\|_{L^{2}}^{2}\right)\left\|e_{u}\right\|_{L^{2}}^{2}, \\
\lambda\left|b\left(e_{u}, \phi, e_{\phi}-\Delta_{h} e_{\phi}\right)\right| \leq & \lambda\left\|e_{u}\right\|_{L^{4}}\|\nabla \phi\|_{L^{4}}\left(\left\|e_{\phi}\right\|_{L^{2}}+\left\|\Delta_{h} e_{\phi}\right\|_{L^{2}}\right) \\
\leq & \frac{1}{16}\left(\nu\left\|\nabla e_{u}\right\|_{L^{2}}^{2}+\lambda \gamma\left\|e_{\phi}\right\|_{L^{2}}^{2}+\left\|\Delta_{h} e_{\phi}\right\|_{L^{2}}^{2}\right) \\
& +\frac{c \lambda^{2}}{\nu \gamma^{2}}\|\phi\|_{H^{1}}^{2}\|\Delta \phi\|_{L^{2}}^{2}\left\|e_{u}\right\|_{L^{2}}^{2}, \\
\lambda\left|b\left(u_{h}, e_{\phi}, e_{\phi}-\Delta_{h} e_{\phi}\right)\right| \leq & \lambda\left\|u_{h}\right\|_{L^{4}}\left\|\nabla e_{\phi}\right\|_{L^{4}}\left(\left\|e_{\phi}\right\|_{L^{2}}+\left\|\Delta_{h} e_{\phi}\right\|_{L^{2}}\right) \\
\leq & \frac{\lambda \gamma}{16}\left(\left\|e_{\phi}\right\|_{L^{2}}^{2}+\left\|\Delta_{h} e_{\phi}\right\|_{L^{2}}^{2}\right) \\
& +c \gamma^{-3}\left\|u_{h}\right\|_{L^{2}}^{2}\left\|\nabla u_{h}\right\|_{L^{2}}^{2} \lambda\left\|e_{\phi}\right\|_{H^{1}}^{2}, \\
\lambda\left|b\left(e_{u}, Q_{h} \phi-\phi, \Delta \phi\right)\right| \leq & \lambda\left\|e_{u}\right\|_{L^{\infty}}\left\|\nabla\left(\phi-Q_{h} \phi\right)\right\|_{L^{2}}\|\Delta \phi\|_{L^{2}} \\
\leq & c \frac{\lambda}{\nu}\|\Delta \phi\|_{L^{2}}^{2}\left\|e_{u}\right\|_{L^{2}}^{2}+c \nu \lambda h^{-2} \| \nabla\left(\phi-Q_{h} \phi \|_{L^{2}}^{2},\right. \\
\lambda\left|b\left(e_{u}, \phi_{h}, \Delta_{h} Q_{h} \phi-\Delta \phi\right)\right| \leq & \lambda\left\|e_{u}\right\|_{L^{4}}\left\|\nabla \phi_{h}\right\|_{L^{4}}\left\|\Delta \phi-\Delta_{h} Q_{h} \phi\right\|_{L^{2}}
\end{aligned}
$$$$
\leq \frac{\nu}{16}\left\|\nabla e_{u}\right\|_{L^{2}}^{2}+\frac{\lambda \gamma}{16}\left\|\Delta \phi-\Delta_{h} Q_{h} \phi\right\|_{L^{2}}^{2}
$$$$
+\frac{c \lambda^{2}}{\nu \gamma^{2}}\left\|\nabla \phi_{h}\right\|_{L^{2}}^{2}\left(\left\|\nabla \phi_{h}\right\|_{L^{2}}^{2}+\left\|\Delta_{h} \phi_{h}\right\|_{L^{2}}^{2}\right)\left\|e_{u}\right\|_{L^{2}}^{2},
$$$$
\lambda\left|b\left(P_{h} u-u, \phi, e_{\phi}-\Delta_{h} e_{\phi}\right)\right| \leq \lambda\left\|P_{h} u-u\right\|_{L^{4}}\|\nabla \phi\|_{L^{4}}\left(\left\|e_{\phi}\right\|_{L^{2}}+\left\|\Delta_{h} e_{\phi}\right\|_{L^{2}}\right)
$$$$
\leq \frac{\lambda \gamma}{16}\left(\left\|e_{\phi}\right\|_{L^{2}}^{2}+\left\|\Delta_{h} e_{\phi}\right\|_{L^{2}}^{2}\right)+\|\Delta \phi\|_{L^{2}}^{2}\left\|u-P_{h} u\right\|_{L^{2}}^{2}
$$$$
+\frac{c \lambda}{\gamma}\|\nabla \phi\|_{L^{2}}^{2}\left\|\nabla\left(u-P_{h} u\right)\right\|_{L^{2}}^{2}
$$$$
\lambda\left|b\left(u_{h}, Q_{h} \phi-\phi, e_{\phi}-\Delta_{h} e_{\phi}\right)\right| \leq \lambda\left\|u_{h}\right\|_{L^{\infty}}\left\|\nabla\left(\phi-Q_{h} \phi\right)\right\|_{L^{2}}\left(\left\|e_{\phi}\right\|_{L^{2}}+\left\|\Delta_{h} e_{\phi}\right\|_{L^{2}}\right)
$$$$
\leq \frac{\lambda \gamma}{16}\left(\left\|e_{\phi}\right\|_{L^{2}}^{2}+\left\|\Delta_{h} e_{\phi}\right\|_{L^{2}}^{2}\right)
$$$$
+\frac{c \lambda}{\gamma} h^{-2}\left\|u_{h}\right\|_{L^{2}}^{2}\left\|\nabla\left(\phi-Q_{h} \phi\right)\right\|_{L^{2}}^{2},
$$$$
\left|\left(\rho_{h} \widetilde{p}-\widetilde{p}, \operatorname{div} e_{u}\right)\right| \leq \frac{\nu}{16}\left\|\nabla e_{u}\right\|_{L^{2}}^{2}+c \nu^{-1}\left\|\widetilde{p}-\rho_{h} \widetilde{p}\right\|_{L^{2}}^{2},
$$$$
\nu\left|\left(\nabla e_{u}, \nabla\left(u-P_{h} u\right)\right)\right| \leq \frac{\nu}{16}\left\|\nabla e_{u}\right\|_{L^{2}}^{2}+c \nu\left\|\nabla\left(u-P_{h} u\right)\right\|_{L^{2}}^{2},
$$$$
\lambda \gamma\left|\left(\nabla e_{\phi}, \nabla\left(Q_{h} \phi-\phi\right)\right)\right| \leq \frac{\lambda \gamma}{16}\left\|\nabla e_{\phi}\right\|_{L^{2}}^{2}+c \lambda \gamma\left\|\nabla\left(\phi-Q_{h} \phi\right)\right\|_{L^{2}}^{2},
$$$$
\lambda \gamma\left|\left(\Delta_{h} e_{\phi}, \Delta_{h} Q_{h} \phi-\Delta \phi\right)\right| \leq \frac{\lambda \gamma}{16}\left\|\Delta_{h} e_{\phi}\right\|_{L^{2}}^{2}+c \lambda \gamma\left\|\Delta \phi-\Delta_{h} Q_{h} \phi\right\|_{L^{2}}^{2} .
$$

Combining these estimates with (3.39) and using (3.2)-(3.5) yield

$$
\begin{aligned}
& \frac{d}{d t}\left(\left\|e_{u}\right\|_{L^{2}}^{2}+\lambda\left\|e_{\phi}\right\|_{H^{1}}^{2}\right)+ \nu\left\|\nabla e_{u}\right\|_{L^{2}}^{2}+\lambda \gamma\left\|\Delta_{h} e_{\phi}\right\|_{L^{2}}^{2} \\
& \leq \varepsilon^{-3} d(t)\left(\left\|e_{u}\right\|_{L^{2}}^{2}+\lambda\left\|e_{\phi}\right\|_{H^{1}}^{2}\right)+c(t) \varepsilon^{-4} h^{2},
\end{aligned}
$$


where

$$
\begin{aligned}
& c(t)=c\left(\lambda \gamma+\nu \lambda+\lambda(1+\lambda)\left(\nu^{-1}+\gamma^{-1}\right)\right)\left(1+\left\|u_{h}\right\|_{L^{2}}^{2}\right)\left(\|\Delta \phi\|_{L^{2}}^{2}+\|\nabla \Delta \phi\|_{L^{2}}^{2}\right) \\
& +c \nu\|\Delta u\|_{L^{2}}^{2}+c \nu^{-1}\|\nabla \widetilde{p}\|_{L^{2}}^{2}+\frac{c \lambda}{\gamma}\left(\|\nabla \phi\|_{L^{2}}^{2}\|\Delta u\|_{L^{2}}^{2}+\|\nabla u\|_{L^{2}}^{2}\|\Delta \phi\|_{L^{2}}^{2}\right) \\
& +c \lambda \gamma\left(1+\|\phi\|_{H^{1}}^{2}+\varepsilon\|\Delta \phi\|_{L^{2}}+\varepsilon\left\|\Delta_{h} \phi_{h}\right\|_{L^{2}}\right)\left(\|\Delta \phi\|_{L^{2}}^{2}+\|\nabla \phi\|_{L^{2}}^{2}\right) \\
& +c \lambda \gamma(F(\phi), 1)^{1 / 2}\left(\left\|\phi^{2}-1\right\|_{H^{1}}+\left\|\phi_{h}^{2}-1\right\|_{H^{1}}\right)\left(\|\Delta \phi\|_{L^{2}}^{2}+\|\nabla \phi\|_{L^{2}}^{2}\right) \\
& +c \frac{\lambda^{2}}{\nu}\left(F\left(\phi_{h}\right), 1\right)\left\|\nabla \phi_{h}\right\|_{L^{2}}\left(\left\|\nabla \phi_{h}\right\|_{L^{2}}+\left\|\Delta_{h} \phi\right\|_{L^{2}}\right)\left(\|\Delta \phi\|_{L^{2}}^{2}+\|\nabla \phi\|_{L^{2}}^{2}\right) \\
& +c \lambda\left(\|\nabla u\|_{L^{2}}^{2}+\left\|\nabla u_{h}\right\|_{L^{2}}^{2}+\left\|\phi_{h}^{2}-1\right\|_{H^{1}}^{2}\right. \\
& \left.+\varepsilon\left\|\nabla \phi_{h}\right\|_{L^{2}}\left(\left\|\nabla \phi_{h}\right\|_{L^{2}}+\left\|\Delta_{h} \phi_{h}\right\|_{L^{2}}\right)\right)\left(\|\Delta \phi\|_{L^{2}}^{2}+\|\nabla \phi\|_{L^{2}}^{2}\right) \\
& +\frac{c \lambda^{2}}{\nu}\left(\left(F\left(\phi_{h}\right), 1\right)^{1 / 2}\left\|\phi_{h}^{2}-1\right\|_{H^{1}}\left\|\nabla \phi_{h}\right\|_{L^{2}}\left(\left\|\nabla \phi_{h}\right\|_{L^{2}}+\left\|\Delta_{h} \phi_{h}\right\|_{L^{2}}\right)\right. \\
& \left.+\left\|\nabla \phi_{h}\right\|_{L^{2}}^{2}\right)\left(1+\left\|\nabla \phi_{h}\right\|_{L^{2}}^{2}\right) \\
& d(t)=c \gamma+c \frac{\lambda}{\gamma}+\frac{c}{\gamma^{3}}\left\|u_{h}\right\|_{L^{2}}^{2}\left\|\nabla u_{h}\right\|_{L^{2}}^{2}+\frac{c \lambda^{2} \varepsilon^{2}}{\nu \gamma^{2}}\left\|\nabla \phi_{h}\right\|_{L^{2}}^{2}\left(\left\|\nabla \phi_{h}\right\|^{2}+\left\|\Delta_{h} \phi_{h}\right\|_{L^{2}}^{2}\right) \\
& +c\left(\frac{\lambda}{\nu}+\frac{\lambda}{\gamma}+\frac{\lambda^{2} \varepsilon^{2}}{\nu \gamma^{2}}\|\phi\|_{H^{1}}^{2}\right)\|\Delta \phi\|_{L^{2}}^{2}+c \gamma\left(\varepsilon^{-1}(F(\phi), 1)^{1 / 2}\left\|\phi^{2}-1\right\|_{H^{1}}\right. \\
& \left.+\varepsilon^{-1}\left(F\left(\phi_{h}\right), 1\right)^{1 / 2}\left\|\phi_{h}^{2}-1\right\|_{H^{1}}\right) \\
& +c \lambda \varepsilon^{-1}\left(F\left(\phi_{h}\right), 1\right)\left\|\nabla \phi_{h}\right\|_{L^{2}}\left(\left\|\nabla \phi_{h}\right\|_{L^{2}}+\left\|\Delta_{h} \phi\right\|_{L^{2}}\right)+c \lambda\left(\|\nabla u\|_{L^{2}}^{2}\right. \\
& \left.+\left\|\nabla u_{h}\right\|_{L^{2}}^{2}+\left\|\phi_{h}^{2}-1\right\|_{H^{1}}^{2}+\left\|\nabla \phi_{h}\right\|_{L^{2}}^{2}+\varepsilon\left\|\nabla \phi_{h}\right\|_{L^{2}}\left\|\Delta_{h} \phi_{h}\right\|_{L^{2}}\right) .
\end{aligned}
$$

Integrating (3.40) from 0 to $t$ and noting $e_{u}(0)=0, e_{\phi}(0)=0$, we obtain

$$
\begin{aligned}
& \left\|e_{u}(t)\right\|_{L^{2}}^{2}+\lambda\left\|e_{\phi}(t)\right\|_{H^{1}}^{2}+\int_{0}^{t}\left(\nu\left\|\nabla e_{u}\right\|_{L^{2}}^{2}+\lambda \gamma\left\|\Delta_{h} e_{\phi}\right\|_{L^{2}}^{2}\right) d s \\
& \leq \varepsilon^{-3} \int_{0}^{t} d(s)\left(\left\|e_{u}\right\|_{L^{2}}^{2}+\lambda\left\|e_{\phi}\right\|_{H^{1}}^{2}\right) d s+\varepsilon^{-4} h^{2} \int_{0}^{T} c(t) d t .
\end{aligned}
$$

By Gronwall's lemma we then get

$$
\begin{aligned}
\left\|e_{u}(t)\right\|_{L^{2}}^{2}+\lambda\left\|e_{\phi}(t)\right\|_{H^{1}}^{2} & +\int_{0}^{t}\left(\nu\left\|\nabla e_{u}\right\|_{L^{2}}^{2}+\lambda \gamma\left\|\Delta_{h} e_{\phi}\right\|_{L^{2}}^{2}\right) d s \\
& \leq \varepsilon^{-4} h^{2} \exp \left(\varepsilon^{-3} \int_{0}^{T} d(t) d t\right) \int_{0}^{T} c(t) d t .
\end{aligned}
$$

From (3.2)-(3.5) it follows that

(3.43) $\left\|u(t)-P_{h} u(t)\right\|_{L^{2}}^{2}+\lambda\left\|\phi(t)-Q_{h} \phi(t)\right\|_{H^{1}}^{2}$

$$
\begin{aligned}
& +\int_{0}^{t}\left(\nu\left\|\nabla\left(u-P_{h} u\right)\right\|_{L^{2}}^{2}+\lambda \gamma\left\|\Delta \phi-\Delta_{h} Q_{h} \phi\right\|_{L^{2}}^{2}\right) d s \\
& \leq c h^{2} \sup _{0 \leq t \leq T}\left\{\|\nabla u(t)\|_{L^{2}}^{2}+\lambda\|\nabla \phi(t)\|_{L^{2}}^{2}+\|\Delta \phi(t)\|_{L^{2}}^{2}\right\} \\
& \quad+c h^{2} \int_{0}^{T}\left(\nu\|\Delta u\|_{L^{2}}^{2}+\lambda \gamma\|\Delta \phi\|_{L^{2}}^{2}+\lambda \gamma\|\nabla \Delta \phi\|_{L^{2}}^{2}\right) d t .
\end{aligned}
$$


Lemmas 2.1, 2.2, and 3.2 imply that there exist some positive constants $c_{j}=$ $c_{j}\left(u_{0}, \phi_{0}, \nu, g, \lambda, \gamma, T\right)$ for $j=1,2,3$ such that

$$
\begin{aligned}
& \int_{0}^{T} d(t) d t \leq c_{1}, \\
& c \sup _{0 \leq t \leq T}\left\{\|\nabla u(t)\|_{L^{2}}^{2}+\lambda\|\nabla \phi(t)\|_{L^{2}}^{2}+\|\Delta \phi(t)\|_{L^{2}}^{2}\right\} \\
& \quad+\int_{0}^{T}\left(c(t)+c \nu\|\Delta u\|_{L^{2}}^{2}+c \lambda \gamma\|\Delta \phi\|_{L^{2}}^{2}+c \lambda \gamma\|\nabla \Delta \phi\|_{L^{2}}^{2}\right) d t \leq c_{2} e^{\varepsilon^{-2} c_{3}} .
\end{aligned}
$$

Combining (3.42) with (3.43), and using (3.44)-(3.45) then yields (3.35).

Finally, integrating (3.37) with $q_{h}=0$ we obtain

$$
\begin{aligned}
& \int_{0}^{t}\left(\rho_{h} \widetilde{p}-\widetilde{p}_{h}, v_{h}\right) d s=\left(u(t)-u_{h}(t), v_{h}\right)-\left(u_{0}^{\varepsilon}-P_{h} u_{0}^{\varepsilon}, v_{h}\right) \\
& \quad+\nu \int_{0}^{t}\left(\nabla\left(u-u_{h}\right), \nabla v_{h}\right) d s-\int_{0}^{t}\left(\widetilde{p}-\rho_{h} \widetilde{p}, \operatorname{div} v_{h}\right) d s \\
& \quad+\lambda \int_{0}^{t} b\left(v_{h}, \phi-\phi_{h}, \Delta \phi\right) d s+\lambda \int_{0}^{t} b\left(v_{h}, \phi_{h}-\phi+\phi, \Delta \phi-\Delta_{h} \phi_{h}\right) d s \\
& \quad-\frac{\lambda}{\varepsilon^{2}} \int_{0}^{t}\left(\left(f(\phi)-Q_{h} f\left(\phi_{h}\right)\right) \nabla \phi+Q_{h} f\left(\phi_{h}\right) \nabla\left(\phi-\phi_{h}\right), v_{h}\right) d s
\end{aligned}
$$

for all $v_{h} \in X_{h}$. Hence, it follows from (3.1), (3.35), and Lemma 2.2 that

$$
\begin{aligned}
&\left\|\int_{0}^{t}\left(\widetilde{p}-\widetilde{p}_{h}\right) d s\right\|_{L^{2}}^{2} \leq 2\left\|\int_{0}^{t}\left(\rho_{h} \widetilde{p}-\widetilde{p}_{h}\right) d s\right\|_{L^{2}}^{2}+2\left\|\int_{0}^{t}\left(\widetilde{p}-\rho_{h} \widetilde{p}\right) d s\right\|_{L^{2}}^{2} \\
& \leq c\left\|\int^{t}\left(\widetilde{p}-\rho_{h} \widetilde{p}\right) d s\right\|_{L^{2}}^{2}+c\left\|u(t)-u_{h}(t)\right\|_{L^{2}}^{2} \\
&+c\left\|u_{0}^{\varepsilon}-P_{h} u_{0}^{\varepsilon}\right\|_{L^{2}}^{2}+c T \nu^{2} \int_{0}^{t}\left\|\nabla\left(u-u_{h}\right)\right\|_{L^{2}}^{2} d s \\
&+c \lambda^{2} \int_{0}^{t}\left(\left\|\nabla\left(\phi-\phi_{h}\right)\right\|_{L^{2}}^{2}\|\Delta \phi\|_{L^{4}}^{2}\right. \\
&\left.+h^{-2}\left\|\nabla\left(\phi-\phi_{h}\right)\right\|_{L^{2}}^{2}\left\|\Delta \phi-\Delta_{h} \phi_{h}\right\|_{L^{2}}^{2}\right) d s \\
&+c \lambda^{2} \int_{0}^{t}\|\nabla \phi\|_{L^{4}}^{2}\|\Delta \phi-\Delta\|_{h} \phi \|_{L^{2}}^{2} d s \\
&+c \lambda^{2} \varepsilon^{-4} \int_{0}^{t}\left\|f(\phi)-Q_{h} f\left(\phi_{h}\right)\right\|_{L^{2}}^{2}\|\nabla \phi\|_{L^{4}}^{2} d s \\
&+c \lambda^{2} \varepsilon^{-4} \int_{0}^{t}\left\|Q_{h} f\left(\phi_{h}\right)\right\|_{L^{4}}^{2}\left\|\nabla\left(\phi-\phi_{h}\right)\right\|_{L^{2}}^{2} d s \\
& \leq c T h^{2} \int^{t}\|\nabla \widetilde{p}\|_{0}^{2} d s+c h^{2}\left\|\nabla u_{0}^{\varepsilon}\right\|_{L^{2}}^{2}+\varepsilon^{-4} C e^{c_{0} \varepsilon^{-3}} h^{2} \\
&+c \lambda^{2} \varepsilon^{-4} \int_{0}^{t}\left(h^{2}\left\|f^{\prime}(\phi) \nabla \phi\right\|_{L^{2}}^{2}\right. \\
&\left.+\left\|Q_{h}\left(\phi^{2}+\phi \phi_{h}+\phi_{h}^{2}-1\right)\left(\phi-\phi_{h}\right)\right\|_{L^{2}}^{2}\right)\|\nabla \phi\|_{L^{4}}^{2} d s \\
&\left.+c \lambda^{2} \varepsilon^{-4} \int_{0}^{t}\left\|Q_{h} f\left(\phi_{h}\right)\right\|_{L^{4}}^{2}\left\|\nabla\left(\phi-\phi_{h}\right)\right\|_{L^{2}}^{2}\right) d s \leq \varepsilon^{-4} C e^{c_{0} \varepsilon^{-3}} h^{2}
\end{aligned}
$$


The proof is complete.

\section{Fully Discrete Finite ElEment approximation AND CONVERGENCE ANALYSIS}

We shall first construct a fully discrete finite element method for approximating the problems (1.1) - 1.5) by discretizing the semi-discrete scheme (3.18)-(3.20) in time. The implicit Euler time-stepping will be used as a prototype scheme for time discretization and for presenting the idea of our convergence analysis. We establish the convergence of the fully discrete numerical solution to the solution of (1.1)-(1.5) as $h, \tau \rightarrow 0$, and to the solution of the sharp interface limiting model (1.6)-(1.11) as $h, \tau, \varepsilon \rightarrow 0$.

4.1. Formulation of fully discrete finite element method. Let $J_{\tau}=\left\{t_{m}\right\}_{m=0}^{M}$ be a quasi-uniform partition of $[0, T]$ of mesh size $\tau:=\frac{T}{M}$, and $d_{t} v^{m}:=\left(v^{m}-\right.$ $\left.v^{m-1}\right) / \tau$. Our fully discrete finite element approximation of problems (1.1)-(1.5) is defined as: find $\left\{\left(u_{h}^{m}, \phi_{h}^{m}, \widetilde{p}_{h}^{m}\right)\right\}_{m=1}^{M} \in X_{h} \times Y_{h} \times M_{h}$ such that for all $\left(v_{h}, \psi_{h}, q_{h}\right) \in$ $X_{h} \times Y_{h} \times M_{h}$

$$
\begin{aligned}
& \left(d_{t} u_{h}^{m}, v_{h}\right)+\nu\left(\nabla u_{h}^{m}, \nabla v_{h}\right)-\left(\widetilde{p}_{h}^{m}, \operatorname{div} v_{h}\right)-\frac{\lambda}{\varepsilon^{2}}\left(Q_{h} f_{h}^{m} \nabla \phi_{h}^{m}, v_{h}\right) \\
& +\lambda\left(\Delta_{h} \phi_{h}^{m} \nabla \phi_{h}^{m}, v_{h}\right)=\left(g^{m}, v_{h}\right), \\
& \left(d_{t} \phi_{h}^{m}, \psi_{h}\right)+\left(u_{h}^{m} \cdot \nabla \phi_{h}^{m}, \psi_{h}\right)+\gamma\left(\nabla \phi_{h}^{m}, \nabla \psi_{h}\right)+\frac{\gamma}{\varepsilon^{2}}\left(f_{h}^{m}, \psi_{h}\right)=0, \\
& \left(\operatorname{div} u_{h}^{m}, q_{h}\right)=0,
\end{aligned}
$$

with the initial conditions $u_{h}^{0}=u_{0 h}:=P_{h} u_{0}^{\varepsilon}$ and $\phi_{h}^{0}=\phi_{0 h}:=Q_{h} \phi_{0}^{\varepsilon}$. Here

$$
g^{m}=\frac{1}{\tau} \int_{t_{m-1}}^{t_{m}} g(t) d t, f_{h}^{m}=\frac{1}{4}\left\{\left|\phi_{h}^{m}\right|^{2}+\left|\phi_{h}^{m-1}\right|^{2}-2\right\}\left\{\phi_{h}^{m}+\phi_{h}^{m-1}\right\} .
$$

Remark 4.1. (a). The $f_{h}^{m}$ factor in the above scheme can be replaced by $\widetilde{f}_{h}^{m}:=$ $\left(\phi_{h}^{m}\right)^{3}-\phi_{h}^{m-1}$. It is not hard to check that the resulted scheme will still satisfy an almost same discrete energy law as that (to be given in the next subsection) satisfied by the above scheme, provided that a mesh constraint on $\tau$ is met (cf. Section 3 of [12]).

(b). Again, the solvability of (4.1)-(4.3) can be verified by using a fixed point argument in finite-dimensional spaces (cf. 27]) and the discrete energy law to be presented in the next subsection.

4.2. Convergence analysis. As demonstrated in the previous section, the standard error estimate technique certainly seems that it will lead to error bounds that depend on $\frac{1}{\varepsilon}$ exponentially! Such an error estimate is not useful as $\varepsilon$ tends to zero. In this subsection, we take a slightly different approach to address the convergence. Instead of proving the convergence by first establishing a rate of convergence (i.e., an error estimate), we shall prove the convergence directly. As expected, the crux for carrying out such a proof is to establish a discrete energy law, which must mimic the basic energy law (2.18), for the proposed numerical method. It should be noted that not every numerical method will meet such a criterion. The goal of this subsection is to prove that the fully discrete finite element method proposed in Section 4.1 indeed is one exception. 
The next lemma is a fully discrete counterpart of Lemma 3.2. which states a discrete energy law for the scheme (4.1)-(4.3).

Lemma 4.1. Let $\left(u_{h}^{m}, \phi_{h}^{m}, \widetilde{p}_{h}^{m}\right)$ solve (4.1)-(4.3). Then there holds that

$$
\begin{aligned}
& \mathcal{E}_{\varepsilon, \lambda}\left(u_{h}^{\ell}, \phi_{h}^{\ell}\right)+\tau \sum_{m=1}^{\ell}\left[\frac{\tau}{2}\left\|d_{t} u_{h}^{m}\right\|_{L^{2}}^{2}+\frac{\tau \lambda}{2}\left\|d_{t} \nabla \phi_{h}^{m}\right\|_{L^{2}}^{2}+\nu\left\|\nabla u_{h}^{m}\right\|_{L^{2}}^{2}\right. \\
& \left.\quad+\lambda \gamma\left\|Q_{h}\left[\Delta_{h} \phi_{h}^{m}-\varepsilon^{-2} f_{h}^{m}\right]\right\|_{L^{2}}^{2}\right]=\tau \sum_{m=1}^{\ell}\left(g^{m}, u_{h}^{m}\right)+\mathcal{E}_{\varepsilon, \lambda}\left(u_{h}^{0}, \phi_{h}^{0}\right)
\end{aligned}
$$

for all $0 \leq \ell \leq M$. Here $\mathcal{E}_{\varepsilon, \lambda}(\cdot, \cdot)$ is defined by (2.19).

Proof. Set

$$
w_{h}^{m}:=Q_{h}\left[\Delta_{h} \phi_{h}^{m}-\varepsilon^{-2} f_{h}^{m}\right]
$$

and rewrite (4.1)-4.2) as

$$
\begin{aligned}
& \left(d_{t} u_{h}^{m}, v_{h}\right)+\nu\left(\nabla u_{h}^{m}, \nabla v_{h}\right)-\left(\widetilde{p}_{h}^{m}, \operatorname{div} v_{h}\right)+\lambda\left(w_{h}^{m} \nabla \phi_{h}^{m}, v_{h}\right)=\left(g^{m}, v_{h}\right), \\
& \left(d_{t} \phi_{h}^{m}, \psi_{h}\right)+\left(u_{h}^{m} \cdot \nabla \phi_{h}^{m}, \psi_{h}\right)-\gamma\left(w_{h}^{m}, \psi_{h}\right)=0 .
\end{aligned}
$$

The desired estimate (4.5) then follows from setting $v_{h}=u_{h}^{m}$ in (4.7), $\psi_{h}=$ $-\lambda w_{h}^{m}$ in (4.8), $q_{h}=\widetilde{p}_{h}^{m}$ in (4.3), testing (4.6) with $d_{t} \phi_{h}^{m}$, adding the resulting equations, using the identities

$$
\begin{aligned}
\left(d_{t} u_{h}^{m}, u_{h}^{m}\right) & =\frac{1}{2}\left\{d_{t}\left\|u_{h}^{m}\right\|_{L^{2}}^{2}+\tau\left\|d_{t} u_{h}^{m}\right\|_{L^{2}}^{2}\right\} \\
\left(d_{t} \nabla u_{h}^{m}, \nabla u_{h}^{m}\right) & =\frac{1}{2}\left\{d_{t}\left\|\nabla u_{h}^{m}\right\|_{L^{2}}^{2}+\tau\left\|d_{t} \nabla u_{h}^{m}\right\|_{L^{2}}^{2}\right\}, \\
\left(d_{t} \phi_{h}^{m}, f_{h}^{m}\right) & =\frac{1}{4} d_{t}\left\|\left(\phi_{h}^{m}\right)^{2}-1\right\|_{L^{2}}^{2},
\end{aligned}
$$

and applying the operator $\tau \sum_{m=1}^{\ell}$ to the combined equation.

Remark 4.2. The above proof actually suggests how to implement the scheme 4.1(4.3) in practice. That is, $w_{h}^{m}$ should be formed in each time step by solving the following equation:

$$
\left(w_{h}^{m}, \chi_{h}\right)=-\left(\nabla \phi_{h}^{m}, \nabla \chi_{h}\right)-\frac{1}{\varepsilon^{2}}\left(f\left(\phi_{h}^{m}\right), \chi_{h}\right) \quad \forall \chi_{h} \in Y_{h} .
$$

In order to take advantage of the wealthy amount of existing Stokes and AllenCahn codes, it is necessary to decouple (4.1) and (4.3) from (4.2) by some iterative procedure. For example, one such iterative procedure is the following three-step fixed point iteration: first, replace $u_{h}^{m}$ by $u_{h}^{m-1}$ in (4.2) and solve the resulting equation to get $\tilde{\phi}_{h}^{m}$, second, solve equation (4.9) with $\tilde{\phi}_{h}^{m}$ in place of $\phi_{h}^{m}$ to get $\tilde{w}_{h}^{m}$, third, solve (4.7) and (4.3) with $\tilde{\phi}_{h}^{m}$ and $\tilde{w}_{h}^{m}$ in place of $\phi_{h}^{m}$ and $w_{h}^{m}$ to get $\left(\tilde{u}_{h}^{m}, \tilde{\tilde{p}}_{h}^{m}\right)$. The iteration is repeated until a stopping criterion is met. Our numerical experiments in Section 5 show that at each time step one or two such iterations are usually sufficient in practice.

The discrete energy law immediately implies the following uniform (in $h$ and $\tau$ ) a priori estimates for $\left(u_{h}^{m}, \phi_{h}^{m}, \widetilde{p}_{h}^{m}\right)$. 
Corollary 4.1. Let $\tau h^{-2} \leq c$ and $\left(u_{h}^{m}, \phi_{h}^{m}, \widetilde{p}_{h}^{m}\right)$ solve (4.1)-(4.3), and suppose that $g \in L^{2}\left(0, T ; L^{2}(\Omega)^{d}\right)$ and that there exists a positive constant $C_{0}$ such that $\mathcal{E}_{\varepsilon, \lambda}\left(u_{0}^{\varepsilon}, \phi_{0}^{\varepsilon}\right) \leq C_{0}$. Then there hold the following estimates:

$$
\begin{aligned}
\max _{0 \leq l \leq M}\left\{\left\|u_{h}^{l}\right\|_{L^{2}}^{2}+\lambda\left\|\nabla \phi_{h}^{l}\right\|_{L^{2}}^{2}+\lambda \varepsilon^{-2}\left(F\left(\phi_{h}^{l}\right), 1\right)\right\} & \leq C, \\
\sum_{m=1}^{M}\left[\left\|u_{h}^{m}-u_{h}^{m-1}\right\|_{L^{2}}^{2}+\lambda\left\|\nabla \phi_{h}^{m}-\nabla \phi_{h}^{m-1}\right\|_{L^{2}}^{2}\right] & \leq C, \\
\sum_{m=1}^{M} \tau\left[\nu\left\|\nabla u_{h}^{m}\right\|_{L^{2}}^{2}+\lambda \gamma\left\|Q_{h}\left[\Delta_{h} \phi_{h}^{m}-\varepsilon^{-2} f_{h}^{m}\right]\right\|_{L^{2}}^{2}\right] & \leq C, \\
\sum_{m=1}^{M} \tau\left\|d_{t} u_{h}^{m}\right\|_{\left(V \cap L^{\infty}\right)^{*}}^{2} & \leq C, \\
\sum_{m=1}^{M} \tau\left\|d_{t} \phi_{h}^{m}\right\|_{L^{\alpha}}^{\alpha} & \leq C, \\
\varepsilon^{-4} \lambda \gamma \sum_{m=1}^{M} \tau\left\|\Delta_{h} \phi_{h}^{m}\right\|_{L^{2}}^{2} & \leq C, \\
\varepsilon^{\frac{d}{3}} \max _{0 \leq l \leq M}\left\|\sum_{m=1}^{l} \tau \tilde{p}_{h}^{m}\right\|_{L^{2}} & \leq C,
\end{aligned}
$$

for any $1 \leq \alpha<\frac{d}{d-1}$.

Proof. (4.10)-(4.12) are the immediate consequences of the discrete energy law (4.1).

To show (4.13), for any $v \in V \cap\left[L^{\infty}(\Omega)\right]^{d}$, setting $v_{h}=P_{h} v$ in (4.7) and using (3.2) we get

$$
\begin{gathered}
\left(d_{t} u_{h}^{m}, v\right)=-\nu\left(\nabla u_{h}^{m}, \nabla P_{h} v\right)-\lambda b\left(P_{h} v-v+v, \phi_{h}^{m}, w_{h}^{m}\right)+\left(g, P_{h} v\right) \\
\leq c \nu\left\|\nabla u_{h}^{m}\right\|_{L^{2}}\|\nabla v\|_{L^{2}}+\lambda\left\|w_{h}^{m}\right\|_{L^{2}}\left\|\nabla \phi_{h}^{m}\right\|_{L^{2}}\|v\|_{L^{\infty}} \\
\quad+\lambda\left\|w_{h}^{m}\right\|_{L^{\infty}}\left\|\nabla \phi_{h}^{m}\right\|_{L^{2}}\left\|P_{h} v-v\right\|_{L^{2}}+c\|g\|_{H^{-1}}\|\nabla v\|_{L^{2}} \\
\leq c \nu\left\|\nabla u_{h}^{m}\right\|_{L^{2}}\|\nabla v\|_{L^{2}}+c \lambda\left\|w_{h}^{m}\right\|_{L^{2}}\left\|\nabla \phi_{h}^{m}\right\|_{L^{2}}\left(\|v\|_{L^{\infty}}+\|\nabla v\|_{L^{2}}\right) \\
\quad+c\|g\|_{H^{-1}}\|\nabla v\|_{L^{2}} .
\end{gathered}
$$

It follows from the above estimate and (4.10)-(4.12) that

$$
\tau \sum_{m=1}^{M}\left\|d_{t} u_{h}^{m}\right\|_{\left(V \cap L^{\infty}\right)^{*}}^{2} \leq C,
$$

hence, 4.13) holds.

Noting that (4.8) is equivalent to

$$
\gamma w_{h}^{m}=d_{t} \phi_{h}^{m}+Q_{h}\left(u_{h}^{m} \cdot \nabla \phi_{h}^{m}\right),
$$

(4.14) then follows from the stability property [6] of the $L^{2}$-projection, (4.10), (4.12), (3.5), and the following inequality:

$$
\left\|u_{h}^{m} \cdot \nabla \phi_{h}^{m}\right\|_{L^{\alpha}}^{\alpha} \leq\left\|u_{h}^{m}\right\|_{L^{s}}^{\alpha}\left\|\nabla \phi_{h}^{m}\right\|_{L^{\frac{s \alpha}{s-\alpha}}}^{\alpha} \quad \forall 1 \leq \alpha<\frac{d}{d-1}, \quad \alpha<s<\frac{2 d}{d-2} .
$$


To show (4.15) and (4.16), from (4.4), (4.6), (4.10)-(4.12), and (3.6) we obtain

$$
\begin{aligned}
\left\|\phi_{h}^{m}\right\|_{L^{2}}^{2} & =\int_{\Omega}\left(\left|\phi_{h}^{m}\right|^{2}-1\right) d x+|\Omega| \leq C, \\
\left\|\Delta_{h} \phi_{h}^{m}\right\|_{L^{2}}^{2} & =\left(w_{h}^{m}, \Delta_{h} \phi_{h}^{m}\right)+\frac{1}{4 \varepsilon}\left(f_{h}^{m}, \Delta_{h} \phi_{h}^{m}\right) \\
& \leq \frac{1}{2}\left\|\Delta_{h} \phi_{h}^{m}\right\|_{L^{2}}^{2}+\left\|w_{h}^{m}\right\|_{L^{2}}^{2}+\varepsilon^{-4}\left\|f_{h}^{m}\right\|_{L^{2}}^{2} \\
& \leq \frac{1}{2}\left\|\Delta_{h} \phi_{h}^{m}\right\|_{L^{2}}^{2}+\left\|w_{h}^{m}\right\|_{L^{2}}^{2}+C \varepsilon^{-4}\left\{\left\|\nabla \phi_{h}^{m-1}\right\|_{L^{2}}^{6}\right. \\
& \left.\quad+\left\|\nabla \phi_{h}^{m}\right\|_{L^{2}}^{6}+\left\|\phi_{h}^{m-1}\right\|_{L^{2}}^{2}+\left\|\phi_{h}^{m}\right\|_{L^{2}}^{2}\right\} \\
& \leq \frac{1}{2}\left\|\Delta_{h} \phi_{h}^{m}\right\|_{L^{2}}^{2}+\left\|w_{h}^{m}\right\|_{L^{2}}^{2}+C \varepsilon^{-4} .
\end{aligned}
$$

Applying the operator $\tau \sum_{m=1}^{l}$ to (4.18) and using (4.12) we get

$$
\lambda \gamma \sum_{m=1}^{l} \tau\left\|\Delta_{h} \phi_{h}\right\|_{L^{2}}^{2} \leq C \varepsilon^{-4},
$$

which gives 4.15).

Finally, applying the operator $\tau \sum_{m=1}^{l}$ to (4.7) yields

$$
\begin{aligned}
\left(\tau \sum_{m=1}^{l} \tilde{p}_{h}^{m}, \operatorname{div} v_{h}\right)= & \left(u_{h}^{l}-u_{h}^{0}, v_{h}\right)+\nu\left(\tau \sum_{m=1}^{l} \nabla u_{h}^{m}, \nabla v_{h}\right) \\
& +\lambda\left(\tau \sum_{m=1}^{l} w_{h}^{m} \nabla \phi_{h}^{m}, v_{h}\right)-\left(\tau \sum_{m=1}^{l} g^{m}, v_{h}\right) .
\end{aligned}
$$

It follows from (3.1), (3.7), (3.9), (4.10), (4.12), and (4.19)-(3.17) that

$$
\begin{gathered}
\left\|\tau \sum_{m=1}^{l} \widetilde{p}_{h}^{m}\right\|_{L^{2}} \leq c\left\|u_{h}^{l}\right\|_{L^{2}}+c\left\|u_{h}^{0}\right\|_{L^{2}}+c \nu T^{\frac{1}{2}}\left(\sum_{m=1}^{l} \tau\left\|\nabla u_{h}^{m}\right\|_{L^{2}}^{2}\right)^{\frac{1}{2}} \\
+c \lambda\left(\sum_{m=1}^{l} \tau\left\|w_{h}^{m}\right\|_{L^{2}}^{2}\right)^{\frac{1}{2}}\left(\sum_{m=1}^{l} \tau\left\|\nabla \phi_{h}^{m}\right\|_{L^{3}}^{2}\right)^{\frac{1}{2}} \\
+c T^{\frac{1}{2}}\left(\int_{0}^{t_{l}}\|g(t)\|_{L^{2}}^{2} d t\right)^{\frac{1}{2}} \\
\leq C+C \lambda\left(\sum_{m=1}^{l} \tau\left\|\nabla \phi_{h}^{m}\right\|_{L^{2}}^{2}\right)^{\frac{6-d}{12}}\left(\sum_{m=1}^{l} \tau\left\|\Delta_{h} \phi_{h}^{m}\right\|_{L^{2}}^{2}\right)^{\frac{d}{12}} \\
\leq C \varepsilon^{-\frac{d}{3}}
\end{gathered}
$$

for $1 \leq l \leq M$. Hence, (4.16) holds. The proof is complete.

Let $\left(U_{\varepsilon, h, \tau}(x, t), \Phi_{\varepsilon, h, \tau}(x, t)\right)$ denote the piecewise linear interpolation (in $t$ ) of the fully discrete solution $\left\{\left(u_{h}^{m}, \phi_{h}^{m}\right)\right\}$, that is,

$$
\begin{aligned}
U_{\varepsilon, h, \tau}(\cdot, t) & :=\frac{t-t_{m-1}}{\tau} u_{h}^{m}(\cdot)+\frac{t_{m}-t}{\tau} u_{h}^{m-1}(\cdot) \quad \forall t \in\left[t_{m-1}, t_{m}\right], \\
\Phi_{\varepsilon, h, \tau}(\cdot, t) & :=\frac{t-t_{m-1}}{\tau} \phi_{h}^{m}(\cdot)+\frac{t_{m}-t}{\tau} \phi_{h}^{m-1}(\cdot) \quad \forall t \in\left[t_{m-1}, t_{m}\right],
\end{aligned}
$$


for $1 \leq m \leq M$, and let $\widetilde{P}_{\varepsilon, h, \tau}(x, t), \bar{U}_{\varepsilon, h, \tau}(x, t)$ and $\bar{\Phi}_{\varepsilon, h, \tau}(x, t)$ denote the piecewise constant extensions of $\left\{\widetilde{p}_{h}^{m}\right\},\left\{u_{h}^{m}\right\}$, and $\left\{\phi_{h}^{m}\right\}$, respectively. Namely,

$$
\begin{array}{lll}
\widetilde{P}_{\varepsilon, h, \tau}(\cdot, t):=\widetilde{p}_{h}^{m} & \forall t \in\left[t_{m-1}, t_{m}\right], & 1 \leq m \leq M, \\
\bar{U}_{\varepsilon, h, \tau}(\cdot, t):=u_{h}^{m} & \forall t \in\left[t_{m-1}, t_{m}\right], & 1 \leq m \leq M, \\
\bar{\Phi}_{\varepsilon, h, \tau}(\cdot, t):=\phi_{h}^{m} & \forall t \in\left[t_{m-1}, t_{m}\right], & 1 \leq m \leq M .
\end{array}
$$

Note that $U_{\varepsilon, h, \tau}(x, t)$ and $\Phi_{\varepsilon, h, \tau}(x, t)$ are continuous piecewise polynomial functions in space and time, and $\widetilde{P}_{\varepsilon, h, \tau}(x, t), \bar{U}_{\varepsilon, h, \tau}(x, t)$, and $\bar{\Phi}_{\varepsilon, h, \tau}(x, t)$ are right continuous at the nodes $\left\{t_{m}\right\}$.

We are now ready to state our main result of this section.

Theorem 4.1. Suppose the assumptions of Corollary 4.1 hold. For each fixed $\varepsilon>0$, let $\left(u_{*}^{\varepsilon}, \phi_{*}^{\varepsilon}, \widetilde{p}_{*}^{\varepsilon}\right)$ denote the unique weak solution of problems (1.1)-(1.5), and $\left\{\left(U_{\varepsilon, h, \tau}, \Phi_{\varepsilon, h, \tau}, \widetilde{P}_{\varepsilon, h, \tau}\right)\right\}$ be defined as above. Then there hold that

$$
\begin{aligned}
& \lim _{h, \tau \rightarrow 0}\left(\left\|U_{\varepsilon, h, \tau}-u_{*}^{\varepsilon}\right\|_{L^{2}\left(L^{2}\right)}+\left\|\nabla \Phi_{\varepsilon, h, \tau}-\nabla \phi_{*}^{\varepsilon}\right\|_{L^{2}\left(L^{2}\right)}\right)=0, \\
& \int_{0}^{t} \widetilde{P}_{\varepsilon, h, \tau} d s \longrightarrow \int_{0}^{t} \widetilde{p}_{*}^{\varepsilon} d s \quad \text { weakly*in } L^{\infty}\left((0, T) ; L^{2}(\Omega)\right) .
\end{aligned}
$$

Proof. Since the proof is long, we divide it into four steps.

Step 1: Extracting convergent subsequences. We first note that the results of Corollary 4.1 imply the following (uniform in $h$ and $\tau$ ) estimates:

$$
\begin{aligned}
& \text { (4.29) }\left\|\bar{U}_{\varepsilon, h, \tau}\right\|_{L^{\infty}\left(L^{2}\right)}+\sqrt{\lambda}\left\|\nabla \bar{\Phi}_{\varepsilon, h, \tau}\right\|_{L^{\infty}\left(L^{2}\right)}+\varepsilon^{-1} \sqrt{\lambda}\left\|\bar{\Phi}_{\varepsilon, h, \tau}^{2}-1\right\|_{L^{\infty}\left(L^{2}\right)} \leq C, \\
& \text { (4.30) } \sqrt{\nu}\left\|\nabla \bar{U}_{\varepsilon, h, \tau}\right\|_{L^{2}\left(L^{2}\right)}+\sqrt{\lambda \gamma}\left\|Q_{h}\left[\Delta_{h} \bar{\Phi}_{\varepsilon, h, \tau}-\varepsilon^{-2} \bar{f}_{\varepsilon, h, \tau}\right]\right\|_{L^{2}\left(L^{2}\right)} \leq C, \\
& \text { (4.31) }\left\|\frac{\partial}{\partial t} U_{\varepsilon, h, \tau}\right\|_{L^{2}\left(\left(V \cap L^{\infty}\right)^{*}\right)} \leq C, \\
& \text { (4.32) }\left\|\frac{\partial}{\partial t} \Phi_{\varepsilon, h, \tau}\right\|_{L^{\alpha}\left(L^{\alpha}\right)} \leq C, \\
& \text { (4.33) } \varepsilon^{2}\left\|\Delta_{h} \bar{\Phi}_{\varepsilon, h, \tau}\right\|_{L^{2}\left(L^{2}\right)} \leq C, \\
& \text { (4.34) } \varepsilon^{\frac{d}{3}} \operatorname{ess~sup~}_{t \in[0, T]}\left\|\int_{0}^{t} \widetilde{P}_{\varepsilon, h, \tau} d s\right\|_{L^{2}} \leq C,
\end{aligned}
$$

where $\bar{f}_{\varepsilon, h, \tau}$ stands for the constant extension (in $t$ ) of $\left\{f_{h}^{m}\right\}$.

Then there exists a convergent subsequence of $\left\{\left(U_{\varepsilon, h, \tau}, \Phi_{\varepsilon, h, \tau}, \widetilde{P}_{\varepsilon, h, \tau}\right)\right\}$ (still denote by the same notation) and a triple $\left(u^{\varepsilon}, \phi^{\varepsilon}, \widetilde{p}^{\varepsilon}\right)$ and another function $w^{\varepsilon}$ such that

$$
\begin{aligned}
& u^{\varepsilon} \in L^{\infty}\left((0, T) ;\left[L^{2}(\Omega)\right]^{d}\right) \cap L^{2}((0, T) ; X) \cap H^{1}\left((0, T) ;\left(V \cap\left[L^{\infty}(\Omega)\right]^{d}\right)^{*}\right), \\
& \phi^{\varepsilon} \in L^{\infty}\left((0, T) ; H^{1}(\Omega)\right) \cap L^{2}\left((0, T) ; H^{2}(\Omega)\right) \cap W^{1, \alpha}\left((0, T) ; L^{\alpha}(\Omega)\right), \\
& \int_{0}^{t} \widetilde{p}^{\varepsilon} d s \in L^{\infty}\left((0, T) ; L^{2}(\Omega)\right), \quad w^{\varepsilon} \in L^{2}\left((0, T) ; L^{2}(\Omega)\right),
\end{aligned}
$$


and

$$
\begin{aligned}
& \bar{U}_{\varepsilon, h, \tau} \stackrel{h, \tau \backslash 0}{\longrightarrow} u^{\varepsilon} \quad \text { weakly } * \text { in } L^{\infty}\left((0, T) ;\left[L^{2}(\Omega)\right]^{d}\right), \\
& \text { strongly in } L^{2}\left((0, T) ;\left[L^{2}(\Omega)\right]^{d}\right) \text {, } \\
& \text { weakly in } L^{2}\left((0, T) ;\left[H^{1}(\Omega)\right]^{d}\right) \text {, } \\
& \bar{\Phi}_{\varepsilon, h, \tau} \stackrel{h, \tau \searrow 0}{\longrightarrow} \phi^{\varepsilon} \quad \text { weakly } * \text { in } L^{\infty}\left((0, T) ; H^{1}(\Omega)\right), \\
& \text { strongly in } L^{2}\left((0, T) ; H^{1}(\Omega)\right) \text {, } \\
& \text { weakly in } W^{1, \alpha}\left((0, T) ; L^{\alpha}(\Omega)\right) \text {, } \\
& \int_{0}^{t} \widetilde{P}_{\varepsilon, h, \tau} d s \stackrel{h, \tau \backslash 0}{\longrightarrow} \int_{0}^{t} \widetilde{p}^{\varepsilon} d s \quad \text { weakly } * \text { in } L^{\infty}\left((0, T) ; L^{2}(\Omega)\right), \\
& \bar{W}_{\varepsilon, h, \tau} \stackrel{h, \tau \backslash 0}{\longrightarrow} w^{\varepsilon} \quad \text { weakly in } L^{2}\left((0, T) ; L^{2}(\Omega)\right),
\end{aligned}
$$

where $\bar{W}_{\varepsilon, h, \tau}:=Q_{h}\left[\Delta_{h} \bar{\Phi}_{\varepsilon, h, \tau}-\varepsilon^{-2} \bar{f}_{\varepsilon, h, \tau}\right]$.

From (4.11) we also have

$$
\begin{aligned}
\left\|U_{\varepsilon, h, \tau}-\bar{U}_{\varepsilon, h, \tau}\right\|_{L^{2}\left(L^{2}\right)}^{2} & =\sum_{m=1}^{M}\left\|u_{h}^{m}-u_{h}^{m-1}\right\|_{L^{2}}^{2} \int_{t_{m-1}}^{t_{m}}\left(\frac{t-t_{m-1}}{\tau}\right)^{2} d t \\
& =\frac{\tau}{3} \sum_{m=1}^{M}\left\|u_{h}^{m}-u_{h}^{m-1}\right\|_{L^{2}}^{2} \stackrel{\tau \searrow 0}{\longrightarrow} 0, \\
\left\|\nabla\left(\Phi_{\varepsilon, h, \tau}-\bar{\Phi}_{\varepsilon, h, \tau}\right)\right\|_{L^{2}\left(L^{2}\right)}^{2} & =\frac{\tau}{3} \sum_{m=1}^{M}\left\|\nabla\left(\phi_{h}^{m}-\phi_{h}^{m-1}\right)\right\|_{L^{2}}^{2} \stackrel{\tau \searrow 0}{\longrightarrow} 0 .
\end{aligned}
$$

Hence, the sequences $\left\{U_{\varepsilon, h, \tau}\right\}$ and $\left\{\bar{U}_{\varepsilon, h, \tau}\right\}$ converge to the same limit as $h, \tau \rightarrow 0$; so do the sequences $\left\{\Phi_{\varepsilon, h, \tau}\right\}$ and $\left\{\bar{\Phi}_{\varepsilon, h, \tau}\right\}$.

Step 2: Passing to the limit. We now want to pass to the limit in (4.1)-(4.3) and show that $\left(u^{\varepsilon}, \phi^{\varepsilon}, \widetilde{p}^{\varepsilon}\right)$ is a weak solution of problems (1.1)-(1.5) with the initial values $u^{\varepsilon}(0)=u_{0}^{\varepsilon}$ and $\phi^{\varepsilon}(0)=\phi_{0}^{\varepsilon}$. To the end, we rewrite (4.1)-(4.3) as

$$
\begin{aligned}
& \left(\left(U_{\varepsilon, h, \tau}\right)_{t}, v_{h}\right)+\nu\left(\nabla \bar{U}_{\varepsilon, h, \tau}, \nabla v_{h}\right)-\left(\widetilde{P}_{\varepsilon, h, \tau}, \operatorname{div} v_{h}\right) \\
& +\lambda\left(\bar{W}_{\varepsilon, h, \tau} \nabla \bar{\Phi}_{\varepsilon, h, \tau}, v_{h}\right)=\left(\bar{g}_{\tau}, v_{h}\right), \\
& \left(\left(\Phi_{\varepsilon, h, \tau}\right)_{t}, \psi_{h}\right)+\left(\bar{U}_{\varepsilon, h, \tau} \cdot \nabla \bar{\Phi}_{\varepsilon, h, \tau}, \psi_{h}\right)-\gamma\left(\bar{W}_{\varepsilon, h, \tau}, \psi_{h}\right)=0, \\
& \left(\operatorname{div} \bar{U}_{\varepsilon, h, \tau}, q_{h}\right)=0 .
\end{aligned}
$$

Here $\bar{g}_{\tau}(t)=g^{m}, t \in\left[t_{m-1}, t_{m}\right]$.

For any $(v, q, \psi) \in\left[H^{2}(\Omega) \cap H_{0}^{1}(\Omega)\right]^{d} \times H^{1}(\Omega) \cap L_{0}^{2}(\Omega) \times H^{2}(\Omega)$, let $\left(v_{h}, q_{h}, \psi_{h}\right)=$ $\left(P_{h} v, \rho_{h} q, Q_{h} \psi\right) \in X_{h} \times M_{h} \times Y_{h}$. 
Multiplying (4.39)-(4.41), respectively, by $\eta(t) \in C^{0}[0, T]$ and integrating in $t$ from 0 to $T$ we get

$$
\begin{gathered}
\int_{0}^{T}\left(\left(U_{\varepsilon, h, \tau}\right)_{t}, v_{h}\right) \eta(t) d t+\int_{0}^{T}\left[\nu\left(\nabla \bar{U}_{\varepsilon, h, \tau}, \nabla v_{h}\right)-\left(\widetilde{P}_{\varepsilon, h, \tau}, \operatorname{div} v_{h}\right)\right] \eta(t) d t \\
+\lambda \int_{0}^{T}\left(\bar{W}_{\varepsilon, h, \tau} \nabla \bar{\Phi}_{\varepsilon, h, \tau}, v_{h}\right) \eta(t) d t=\int_{0}^{T}\left(\bar{g}_{\tau}, v_{h}\right) \eta(t) d t \\
\begin{array}{r}
\int_{0}^{T}\left(\left(\Phi_{\varepsilon, h, \tau}\right)_{t}, \psi_{h}\right) \eta(t) d t+\int_{0}^{T}\left[\left(\bar{U}_{\varepsilon, h, \tau} \cdot \nabla \bar{\Phi}_{\varepsilon, h, \tau}, \psi_{h}\right)\right. \\
\left.-\gamma\left(\bar{W}_{\varepsilon, h, \tau}, \psi_{h}\right)\right] \eta(t) d t=0 \\
\int_{0}^{T}\left(\operatorname{div} \bar{U}_{\varepsilon, h, \tau}, q_{h}\right) \eta(t) d t=0 .
\end{array}
\end{gathered}
$$

Setting $h, \tau \rightarrow 0$ in (4.42)-(4.44) and using (4.35)-(4.38) we get

$$
\begin{gathered}
\int_{0}^{T}\left\langle u_{t}^{\varepsilon}, v\right\rangle \eta(t) d t+\nu \int_{0}^{T}\left(\nabla u^{\varepsilon}, \nabla v\right) \eta(t) d t-\int_{0}^{T}\left(\widetilde{p}^{\varepsilon}, \operatorname{div} v\right) \eta(t) d t \\
+\lambda \int_{0}^{T}\left(w^{\varepsilon} \nabla \phi^{\varepsilon}, v\right) \eta(t) d t=\int_{0}^{T}(g, v) \eta(t) d t \\
\int_{0}^{T}\left\langle\phi_{t}^{\varepsilon}, \psi\right\rangle \eta(t) d t+\int_{0}^{t}\left(u^{\varepsilon} \cdot \nabla \phi^{\varepsilon}, \psi\right) \eta(t) d t-\gamma \int_{0}^{T}\left(w^{\varepsilon}, \psi\right) \eta(t) d t=0, \\
\int_{0}^{T}\left(\operatorname{div} u^{\varepsilon}, q\right) \eta(t) d t=0,
\end{gathered}
$$

for all $\eta(t) \in C^{0}[0, T]$ and $(v, q, \psi) \in\left[H^{2}(\Omega) \cap H_{0}^{1}(\Omega)\right]^{d} \times H^{1}(\Omega) \cap L_{0}^{2}(\Omega) \times H^{2}(\Omega)$.

Since $C^{0}[0, T]$ is dense in $L^{2}(0, T)$ and $\left[H^{2}(\Omega) \cap H_{0}^{1}(\Omega)\right]^{d}$ is dense in $\left[H_{0}^{1}(\Omega)\right]^{d}$, $H^{1}(\Omega) \cap L_{0}^{2}(\Omega)$ is dense in $L_{0}^{2}(\Omega)$ and $H^{2}(\Omega)$ is dense in $H^{1}(\Omega)$, we deduce from (4.45) -4.47) that

$$
\begin{aligned}
& \left\langle u_{t}^{\varepsilon}, v\right\rangle+\nu\left(\nabla u^{\varepsilon}, \nabla v\right)-\left(\widetilde{p}^{\varepsilon}, \operatorname{div} v\right)+\lambda\left(w^{\varepsilon} \nabla \phi^{\varepsilon}, v\right)=(g, v), \\
& \left\langle\phi_{t}^{\varepsilon}, \psi\right\rangle+\left(u^{\varepsilon} \cdot \nabla \phi^{\varepsilon}, \psi\right)-\gamma\left(w^{\varepsilon}, \psi\right)=0, \\
& \left(\operatorname{div} u^{\varepsilon}, q\right)=0
\end{aligned}
$$

for all $(v, q, \psi) \in\left[H_{0}^{1}(\Omega)\right]^{d} \times L_{0}^{2}(\Omega) \times H^{1}(\Omega)$.

It remains to prove $u^{\varepsilon}(0)=u_{0}^{\varepsilon}$ and $\phi^{\varepsilon}(0)=\phi_{0}^{\varepsilon}$. To the end, replacing $\eta(t)$ in (4.45) and (4.46) by $\eta_{T}(t)=-\frac{t}{T}+1$ and integrating by parts with respect to $t$ in the first terms of (4.45) and (4.46) we get

$$
\begin{aligned}
\int_{0}^{T} \frac{1}{T}\left(u^{\varepsilon}, v\right) d t & +\nu \int_{0}^{T}\left(\nabla u^{\varepsilon}, \nabla v\right) \eta_{T}(t) d t-\int_{0}^{T}\left(\widetilde{p}^{\varepsilon}, \operatorname{div} v\right) \eta_{T}(t) d t \\
& +\lambda \int_{0}^{T}\left(w^{\varepsilon} \nabla \phi^{\varepsilon}, v\right) \eta(t) d t=\left(u^{\varepsilon}(0), v\right)+\int_{0}^{T}(g, v) \eta_{T}(t) d t \\
\int_{0}^{T} \frac{1}{T}\left(\phi^{\varepsilon}, \psi\right) d t & +\int_{0}^{t}\left[\left(u^{\varepsilon} \cdot \nabla \phi^{\varepsilon}, \psi\right)-\gamma\left(w^{\varepsilon}, \psi\right)\right] \eta_{T}(t) d t=\left(\phi^{\varepsilon}(0), \psi\right)
\end{aligned}
$$


Repeating the same argument in (4.42)-4.43) yields

$$
\begin{gathered}
\text { (4.53) } \int_{0}^{T} \frac{1}{T}\left(U_{\varepsilon, h, \tau}, v_{h}\right) d t+\int_{0}^{T}\left[\nu\left(\nabla \bar{U}_{\varepsilon, h, \tau}, \nabla v_{h}\right)-\left(\widetilde{P}_{\varepsilon, h, \tau}, \operatorname{div} v_{h}\right)\right] \eta_{T}(t) d t \\
+\lambda \int_{0}^{T}\left(\bar{W}_{\varepsilon, h, \tau} \nabla \bar{\Phi}_{\varepsilon, h, \tau}, v_{h}\right) \eta_{T}(t) d t=\left(P_{h} u_{0}^{\varepsilon}, v_{h}\right)+\int_{0}^{T}\left(\bar{g}_{\tau}, v_{h}\right) \eta_{T}(t) d t \\
\begin{array}{c}
\text { (4.54) } \int_{0}^{T} \frac{1}{T}\left(\Phi_{\varepsilon, h, \tau}, \psi_{h}\right) d t+\int_{0}^{T}\left(\bar{U}_{\varepsilon, h, \tau} \cdot \nabla \bar{\Phi}_{\varepsilon, h, \tau}, \psi_{h}\right) \eta_{T}(t) d t \\
-\gamma \int_{0}^{T}\left(\bar{W}_{\varepsilon, h, \tau}, \psi_{h}\right) \eta_{T}(t) d t=\left(Q_{h} \phi_{0}^{\varepsilon}, \psi_{h}\right) .
\end{array}
\end{gathered}
$$

Thus, taking the limit $(h, \tau) \rightarrow(0,0)$ in (4.53) -4.54 ) we obtain

$$
\begin{aligned}
& \int_{0}^{T} \frac{1}{T}\left(u^{\varepsilon}, v\right) d t+\nu \int_{0}^{T}\left(\nabla u^{\varepsilon}, \nabla v\right) \eta_{T}(t) d t-\int_{0}^{T}\left(\widetilde{p}^{\varepsilon}, \operatorname{div} v\right) \eta_{T}(t) d t \\
&+\lambda \int_{0}^{T}\left(w^{\varepsilon} \nabla \phi^{\varepsilon}, v\right) \eta_{T}(t) d t=\left(u_{0}^{\varepsilon}, v\right)+\int_{0}^{T}(g, v) \eta_{T}(t) d t, \\
& \int_{0}^{T} \frac{1}{T}\left(\phi^{\varepsilon}, \psi\right) d t+\int_{0}^{T}\left(u^{\varepsilon} \cdot \nabla \phi^{\varepsilon}, \psi\right) \eta_{T}(t) d t \\
&-\gamma \int_{0}^{T}\left(w^{\varepsilon}, \psi\right) \eta_{T}(t) d t=\left(\phi_{0}^{\varepsilon}, \psi\right) .
\end{aligned}
$$

Comparing (4.51)-(4.52) with (4.55)-(4.56) yields $u^{\varepsilon}(0)=u_{0}^{\varepsilon}$ and $\phi^{\varepsilon}(0)=\phi_{0}^{\varepsilon}$.

Step 3: Identifying $w^{\varepsilon}$. It follows from the definition of $\bar{W}_{\varepsilon, h, \tau}$ in (4.38) that

$$
\begin{aligned}
\int_{0}^{T}\left(\bar{W}_{\varepsilon, h, \tau}, \psi_{h}\right) \eta(t) d t & =\int_{0}^{T}\left(\Delta_{h} \bar{\Phi}_{\varepsilon, h, \tau}-\varepsilon^{-2} \bar{f}_{\varepsilon, h, \tau}, \psi_{h}\right) \eta(t) d t \\
& =-\int_{0}^{T}\left[\left(\nabla \bar{\Phi}_{\varepsilon, h, \tau}, \nabla \psi_{h}\right)-\varepsilon^{-2}\left(\bar{f}_{\varepsilon, h, \tau}, \psi_{h}\right)\right] \eta(t) d t
\end{aligned}
$$

for all $\psi_{h} \in Y_{h}$ and $\eta(t) \in C[0, T]$.

Letting $h, \tau \rightarrow 0$ and using (4.36), (4.38), the definition of $f_{h}^{m}$, and the above density argument we have

$$
\int_{0}^{T}\left(w^{\varepsilon}, \psi\right) \eta(t) d t=-\int_{0}^{T}\left(\nabla \phi^{\varepsilon}, \nabla \psi\right) \eta(t) d t-\frac{1}{\varepsilon^{2}} \int_{0}^{T}\left(f\left(\phi^{\varepsilon}\right), \psi\right) \eta(t) d t
$$

for all $\psi \in H^{1}(\Omega), \eta(t) \in C[0, T]$, which implies that

$$
\begin{aligned}
\left(w^{\varepsilon}, \psi\right) & =-\left(\nabla \phi^{\varepsilon}, \nabla \psi\right)-\frac{1}{\varepsilon^{2}}\left(f\left(\phi^{\varepsilon}\right), \psi\right) \quad \forall \psi \in H^{1}(\Omega), \\
\frac{\partial \phi^{\varepsilon}}{\partial n} & =0 \quad \text { on } \partial \Omega .
\end{aligned}
$$

Hence, $\left(u^{\varepsilon}, \phi^{\varepsilon}, \widetilde{p}^{\varepsilon}\right)$ is a weak solution of (1.1)-(1.5).

Step 4: Finishing up. We have shown above that $\left\{\left(U_{\varepsilon, h, \tau}, \Phi_{\varepsilon, h, \tau}, \widetilde{P}_{\varepsilon, h, \tau}\right)\right\}$ has a convergent subsequence and its limit $\left(u^{\varepsilon}, \phi^{\varepsilon}, \widetilde{p}^{\varepsilon}\right)$ is a weak solution of (1.1)(1.5). By the uniqueness, we have $u^{\varepsilon}=u_{*}^{\varepsilon}, \phi^{\varepsilon}=\phi_{*}^{\varepsilon}$ and $\widetilde{p}^{\varepsilon}=\widetilde{p}_{*}^{\varepsilon}$. Moreover, the proof also implies that the limit of every convergent subsequence of $\left\{\left(U_{\varepsilon, h, \tau}, \Phi_{\varepsilon, h, \tau}, \widetilde{P}_{\varepsilon, h, \tau}\right)\right\}$ must be a weak solution of (1.1)-(1.5). Hence, the whole 
sequence $\left\{\left(U_{\varepsilon, h, \tau}, \Phi_{\varepsilon, h, \tau}, \widetilde{P}_{\varepsilon, h, \tau}\right)\right\}$ must converge to the unique weak solution $\left(u_{*}^{\varepsilon}, \phi_{*}^{\varepsilon}\right.$, $\left.\tilde{p}_{*}^{\varepsilon}\right)$. The proof is complete.

An immediate consequence of Theorem 4.1 and the convergence result of 24 ] (see also Theorem 3.3 of [25]) is the following convergence theorem.

Theorem 4.2. Assume that the sharp interface problems (1.6)-(1.11) have a unique regular solution $\left(u_{*}, p_{*}\right)$. Under the assumptions of Theorem 4.1 there hold

$$
\begin{aligned}
& \lim _{\varepsilon \rightarrow 0} \lim _{h, \tau \rightarrow 0}\left\|U_{\varepsilon, h, \tau}-u_{*}\right\|_{L^{2}\left(L^{2}\right)}=0, \\
& \Phi_{\varepsilon, h, \tau} \stackrel{\varepsilon, h, \tau \backslash 0}{\longrightarrow} \pm 1 \quad \text { a.e. in } \Omega_{t}^{ \pm} \times(0, T) .
\end{aligned}
$$

Here $\Omega_{t}^{+}$and $\Omega_{t}^{-}$denote the outside and the inside of $\Gamma_{t}$ in $\Omega$ at time $t$, respectively.

Remark 4.3. (a). There is no information above the convergence of $\widetilde{P}_{\varepsilon, h, \tau}$ to $p_{*}$ due to the fact that the estimate (4.16) depends on $\varepsilon$, hence, the convergence of (4.28) is not uniform in $\varepsilon$.

(b). Essentially, the convergence result of Theorem 4.1 guarantees that the numerical solution $\left(U_{\varepsilon, h, \tau}, \Phi_{\varepsilon, h, \tau}, \widetilde{P}_{\varepsilon, h, \tau}\right)$ enjoys the same kind convergence to the solution $\left(u_{*}, p_{*}\right)$ of the limiting problem (1.6)-(1.11) as the phase field solution $\left(u_{*}^{\varepsilon}, \phi_{*}^{\varepsilon}, \widetilde{p}_{*}^{\varepsilon}\right)$ of (1.1) -1.5$)$ does.

\section{Numerical EXPERIMENTS}

In this section we provide some 2-D numerical experiments to gauge the fully discrete finite element method developed in the previous sections. In addition, our numerical results reveal some interesting features such as shrinking, splitting, and tangential vibration of fluid interfaces governed by the phase field model (1.1)-(1.5). In all numerical experiments to be given in the following, we choose $\Omega=[-0.5,0.5]^{2}$, $\varepsilon=10^{-2}, \nu=\lambda=\gamma=0.1$, and $u_{0}^{\varepsilon} \equiv 0$, while the initial condition for $\phi$ is specified in each test. Also, in order to resolve the diffuse interface, we use $\tau=10^{-5}$ and unstructured spatial meshes in all experiments.

Test 1. In this test, we take the following initial condition for $\phi$ :

$$
\phi_{0}^{\varepsilon}(x)=\tanh \left(\frac{x_{1}^{2}}{0.01}+\frac{x_{2}^{2}}{0.0225}-1\right) .
$$

Note that the zero level set of $\phi_{0}^{\varepsilon}$, which gives the initial fluid interface, is the ellipse

$\frac{x_{1}^{2}}{0.01}+\frac{x_{2}^{2}}{0.0225}=1$. Hence, we have the situation of one elliptical fluid bubble inside another fluid.

Figure 1 shows snapshots of color and zero-level set plots of the computed phase function $\phi_{h}^{m}$ at six time steps. In the figure, the interior of the ellipse is where $\phi_{h}^{m}=1$, the exterior of the ellipse is where $\phi_{h}^{m}=-1$, and the black curve represents the zero-level set of the computed phase function. We note that the elliptical bubble quickly deforms into a circular bubble, while the size of the bubble shrinks. The bubble eventually shrinks to zero (i.e., it eventually disappears), due to the dissipative mechanism of the phase field model (1.1)-(1.5) (cf. Lemma 2.1). In this test, the fluid bubble disappears at $t=7.5 \times 10^{-4}$. We also note that the surface of the bubble shows a tangential vibration before it becomes a circular bubble. This tangential vibration is due to the so-called T-modes of the spheric normal modes 
(cf. 25] and the references therein). Hence, this shows that our model captures this important special effect of the surface tension. We also remark that the interface (zero-level set) movement is very similar to that of the zero-level set of the solution to the Allen-Cahn equation (the equation obtained by setting $u \equiv 0$ in (1.2)) (cf. [14]). As expected, here the zero-level set is pushed slightly off the center by the fluid flow (through the convective term $u \cdot \nabla \phi$ ) while it is shrinking.

Figure 2 displays snapshots of the arrow and streamline plots of the computed velocity field $u_{h}^{m}$ at six time steps. The ellipse in the center of each snapshot stands for the initial fluid interface (i.e., the zero-level set of $\phi_{0}^{\varepsilon}$ ). We note that fluid vertices are formed shortly after the initial time step, and more vertices are produced as time goes on.
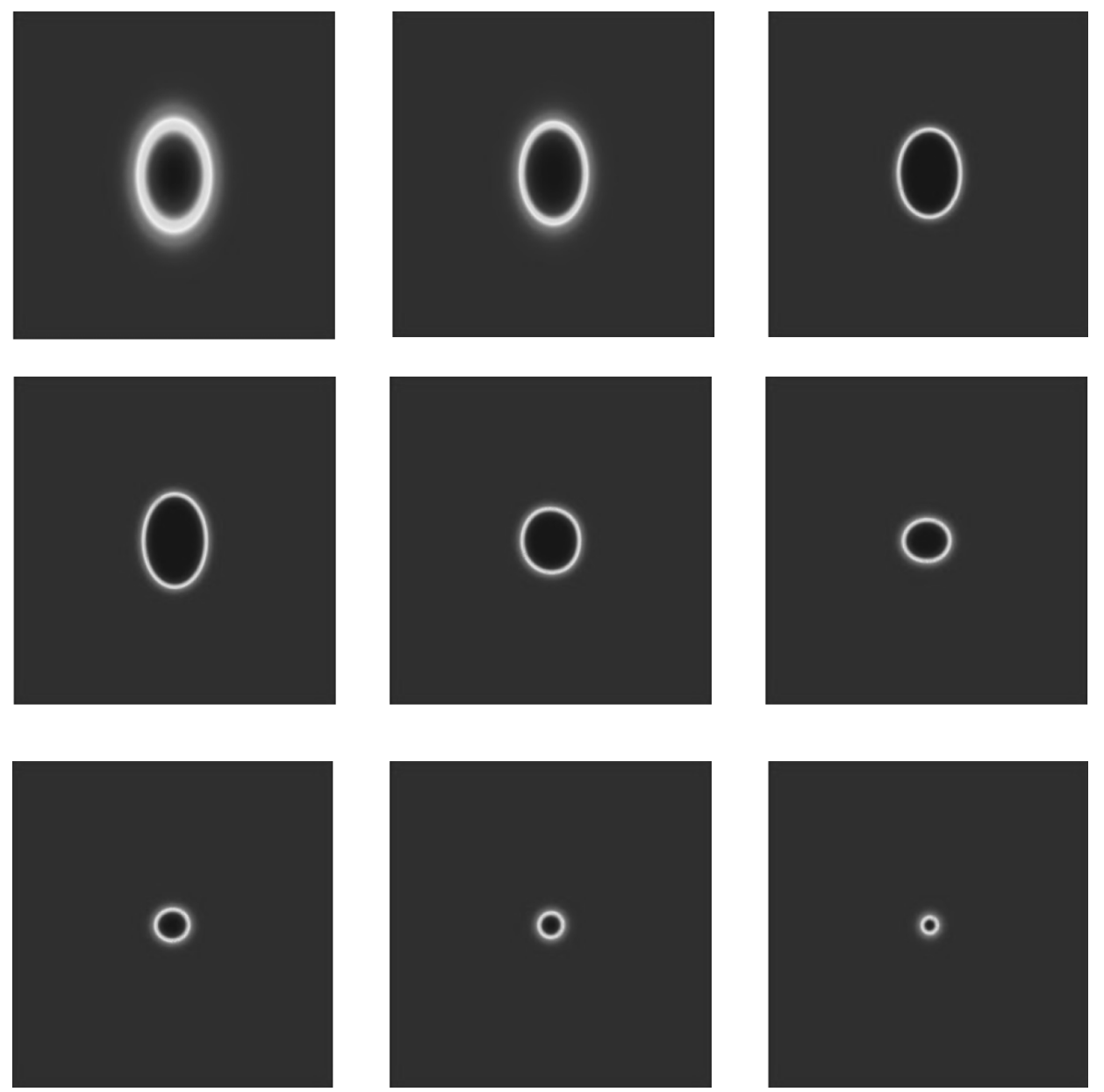

Figure 1. Color and zero-level set plots of computed phase function $\phi_{h}^{m}$ at $t_{m}=10^{-5}, 10^{-4}, 5 \times 10^{-4}, 10^{-3}, 3 \times 10^{-3}, 5 \times 10^{-3}, 6 \times$ $10^{-3}, 6.5 \times 10^{-3}, 6.9 \times 10^{-3}$. The graphs are arranged row-wise. 

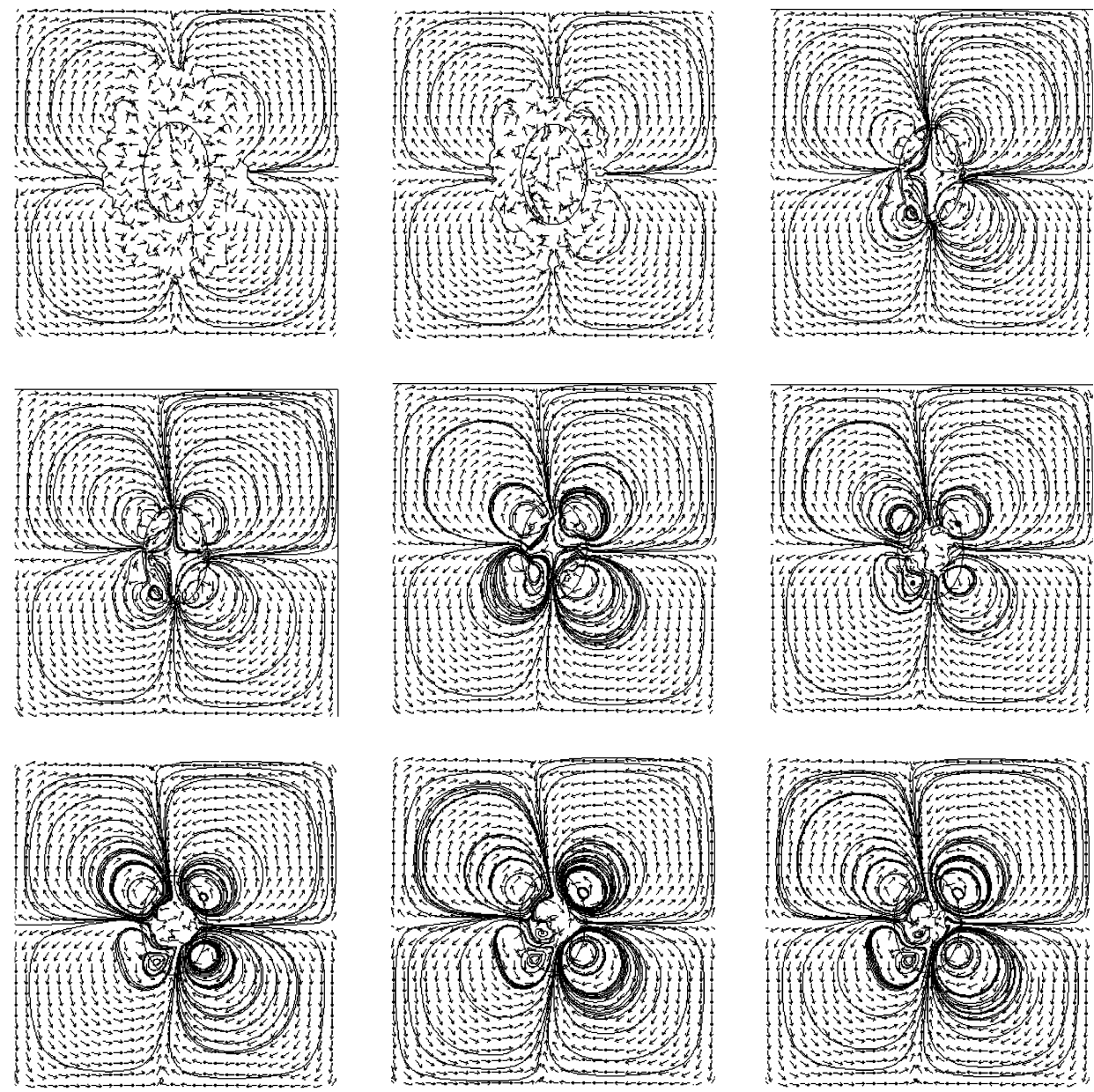

Figure 2. Arrow and streamline plots of computed velocity field $u_{h}^{m}$ at $t_{m}=10^{-5}, 10^{-4}, 5 \times 10^{-4}, 10^{-3}, 3 \times 10^{-3}, 5 \times 10^{-3}, 6 \times$ $10^{-3}, 6.5 \times 10^{-3}, 6.9 \times 10^{-3}$. The graphs are arranged row-wise.

Test 2. In this test, the initial profile of the phase function is taken as

$$
\phi_{0}^{\varepsilon}(x)=\tanh \left(\frac{1}{\varepsilon}\left(\frac{x_{1}^{2}}{0.01}+\frac{x_{2}^{2}}{0.04}-1\right)\left(\frac{x_{1}^{2}}{0.04}+\frac{x_{2}^{2}}{0.01}-1\right)\right) .
$$

Note that the zero level set of $\phi_{0}^{\varepsilon}$, which gives the initial fluid interface, is the union of the following two intersecting ellipses: $\frac{x_{1}^{2}}{0.01}+\frac{x_{2}^{2}}{0.04}=1$ and $\frac{x_{1}^{2}}{0.04}+\frac{x_{2}^{2}}{0.01}=1$, which enclose four bullet-head-like bubbles inside a fluid.

Figure 3 shows snapshots of color and zero-level set plots of the computed phase function $\phi_{h}^{m}$ at six time steps. Again, the interior of the bullet-head bubbles is where $\phi_{h}^{m}=1$, the exterior of the bullet-head bubbles is where $\phi_{h}^{m}=-1$, and the black curve represents the zero-level set of the computed phase function. In this test, the fluid bubble disappears at $t=1.89 \times 10^{-3}$. We also remark that the interface (zero-level set) movement is very similar to that of the zero-level set of the solution to the Allen-Cahn equation (the equation obtained by setting $u \equiv 0$ 
in (1.2) ) (cf. [14]). As expected, here the zero-level set is pushed slightly off the center by the fluid flow (through the convective term $u \cdot \nabla \phi$ ) while it is shrinking. Another noticeable difference is that, unlike the dynamics of the zero-level set of the solution to the Allen-Cahn equation, here the four bullet-head-like bubbles seems to shrink at slightly different speed and the bottom bubble disappears a couple of time steps earlier than the middle two, which in turn vanishes a couple of time steps earlier than the top bubble. We think that this behavior is caused by the fluid flow through the convective term $u \cdot \nabla \phi$.

Figure 4 displays snapshots of the arrow and streamline plots of the computed velocity field $u_{h}^{m}$ at six time steps. The ellipses in the center of each snapshot stand for the initial fluid interface (i.e., the zero-level set of $\phi_{0}^{\varepsilon}$ ). Again, we note that fluid vertices are formed shortly after the initial time step, and more vertices are produced as time goes on.
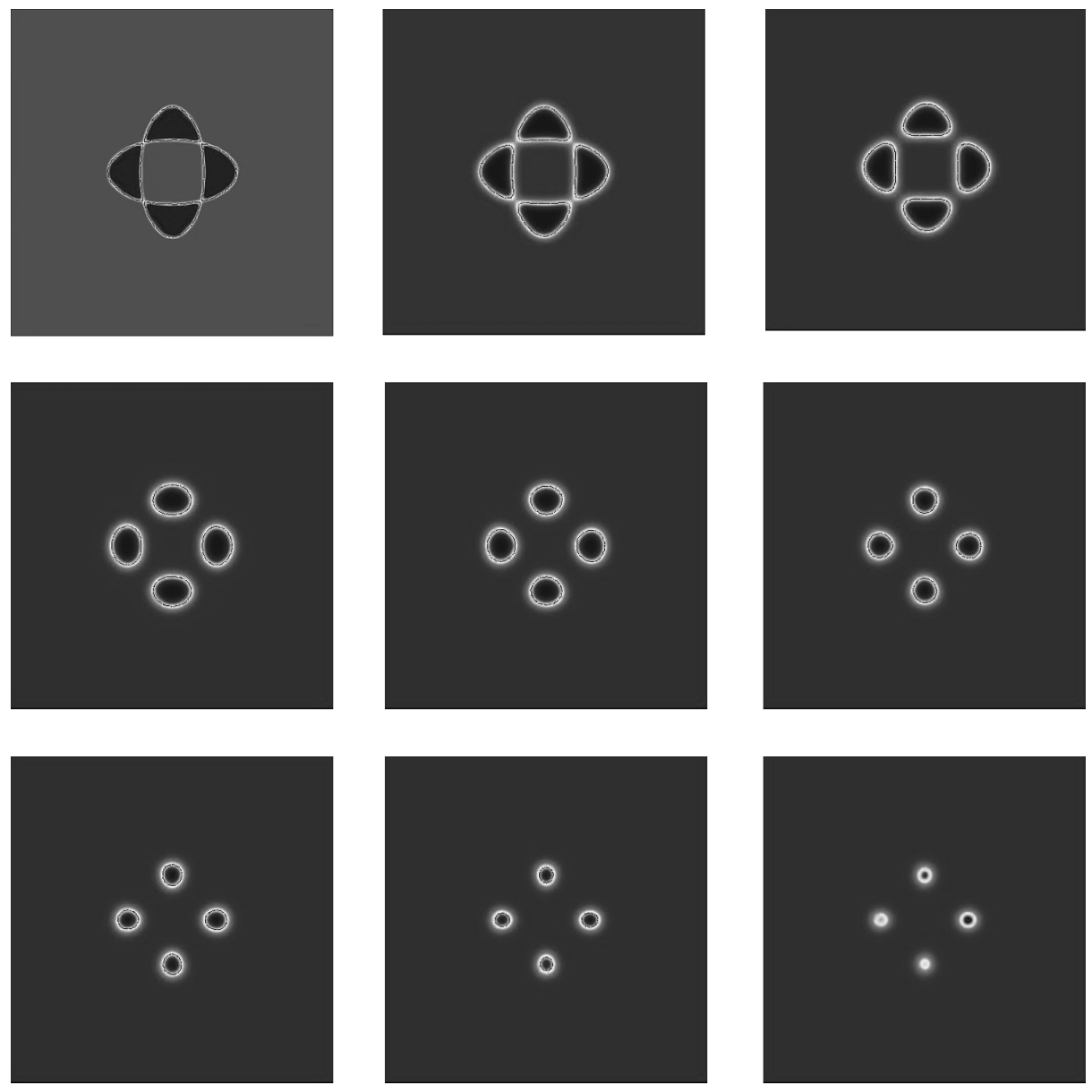

Figure 3. Color and zero-level set plots of computed phase function $\phi_{h}^{m}$ at $t_{m}=10^{-5}, 10^{-4}, 3 \times 10^{-4}, 7 \times 10^{-4}, 10 \times 10^{-4}, 13 \times$ $10^{-4}, 15 \times 10^{-4}, 17 \times 10^{-4}, 1.88 \times 10^{-3}$. The graphs are arranged row-wise. 

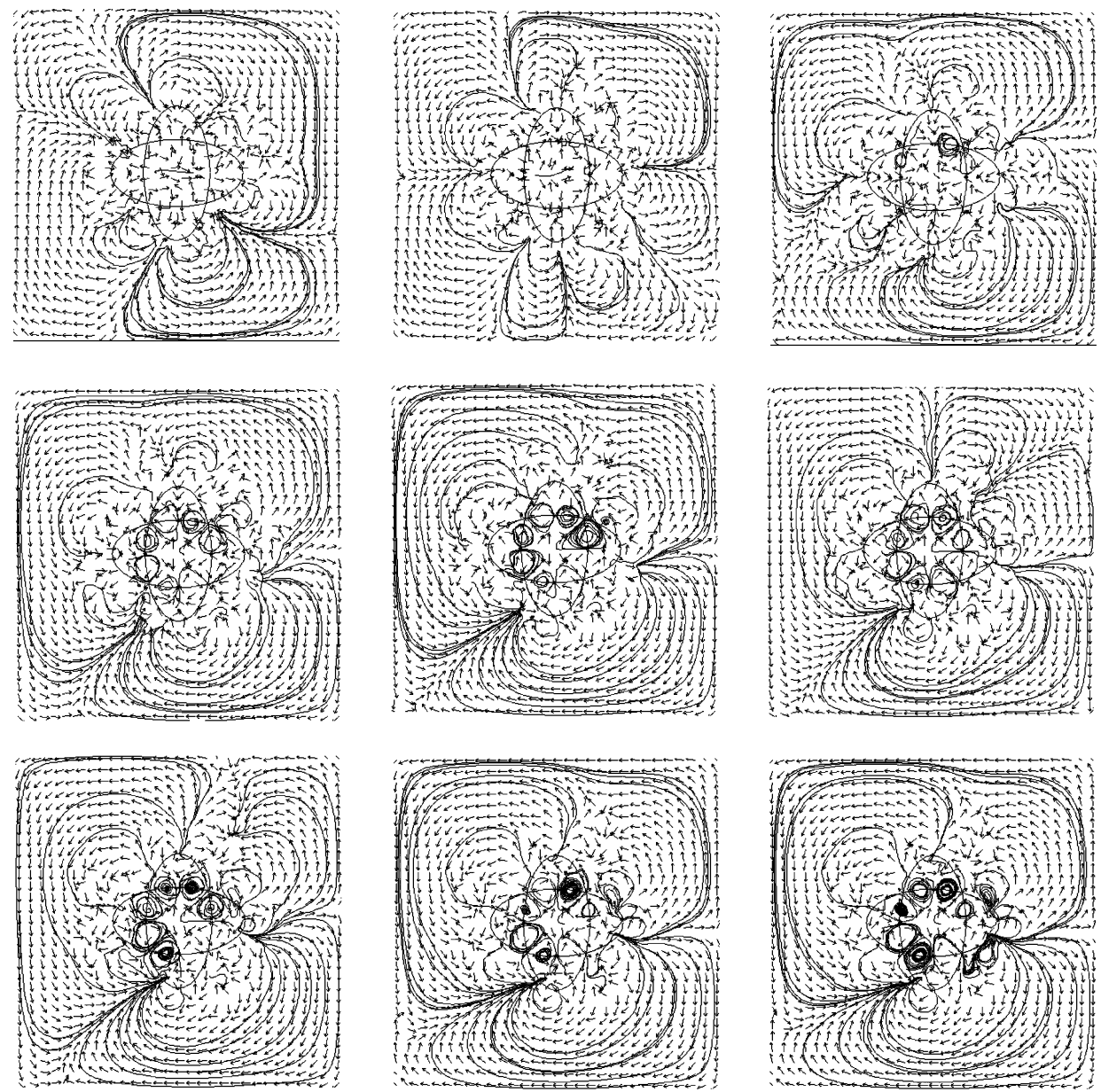

Figure 4. Arrow and streamline plots of computed velocity field $u_{h}^{m}$ at $t_{m}=10^{-5}, 10^{-4}, 4 \times 10^{-4}, 7 \times 10^{-4}, 10^{-3}, 1.3 \times 10^{-3}, 1.6 \times$ $10^{-3}, 1.8 \times 10^{-3}, 1.88 \times 10^{-3}$. The graphs are arranged row-wise.

\section{REFERENCES}

[1] R. A. Adams, Sobolev Spaces, Academic Press, New York, 1975. MR0450957 (56:9247)

[2] I. Babuska, J. Osborn and J. Pitkaranta, Analysis of mixed methods using mesh dependent norms, Math. Comp., 35(1980), pp. 1039-1062. MR0583486 (81m:65166)

[3] R. Becker, X. Feng And A. Prohl, Finite element methods for the Ericksen-Leslie model of flow of nematic liquid crystals (submitted).

[4] M. Bercovier And O. Pironneau, Error estimates for finite element solution of the Stokes problem in the primitive variables, Numer. Math., 33(1979), pp. 211-224. MR0549450 (81g:65145)

[5] J. Bear, Dynamics of Fluids in Porous Media, Dover Publications, Inc., New York, 1972.

[6] J. H. Bramble and J. Xu, Some estimates for a weighted $L^{2}$ projection, Math. Comp., 56(1991), pp. 463-576. MR.1066830 (91k:65140)

[7] F. Brezzi And M. Fortin, Mixed and Hybrid Finite Element Methods, Springer-Verlag, New York, 1992. MR1115205(92d:65187) 
[8] G. Caginalp, An analysis of a phase field model of a free boundary, Arch. Rational Mech. Anal., 92(1986), pp. 205-245. MR0816623 (87c:80011)

[9] P. G. Ciarlet, The Finite Element Method for Elliptic Problems, North-Holland, Amsterdam, 1978. MR0520174 (58:25001)

[10] I. V. Denisova AND V. A. SOlONNIKov, Solvability of a linearized problem on the motion of a drop in a fluid flow, Translation in J. Soviet Math. 56(1991), pp. 2309-2316. MR1031984 (90m:35153)

[11] D. A. Edwards, H. Brenner and D. T. Wasan, Interfacial Transport Process and Rheology, Butterworths/Heinemann, London, 1991.

[12] X. Feng And A. Prohl, Numerical Analysis of the Allen-Cahn equation and approximation for mean curvature flows, Numer. Math., 94(2003), pp. 33-65. MR1971212 (2004e:65111)

[13] X. Feng And A. Prohl, Analysis of a fully discrete finite element method for the phase field model and approximation of its sharp interface limits, Math. Comp., 37(2003), pp. 541-567. MR2028419 (2005b:74115)

[14] X. FENG AND H. WU, A posteriori error estimates and an adaptive finite element algorithm for the Allen-Cahn equation and the mean curvature flow, J. Scient. Comput., 24(2005). MR2221163

[15] P. FIFE, Dynamics of Internal Layers and Diffusive Interfaces, SIAM, Philadelphia, PA, 1988. MR0981594 (90c:80012)

[16] G. Fix, Phase field method for free boundary problems, in Free Boundary Problems (A. Fasano and M. Primicerio editors), Pitman, London, 1983, pp. 580-589.

[17] D. Gilbarg and N. S. Trudinger, Elliptic Partial Differential Equations of Second Order, Second Edition, Springer, New York, 2000.

[18] V. Girault and P. A. Raviart, Finite Element Method for Navier-Stokes Equations: Theory and Algorithms, Springer-Verlag, Berlin, Heidelberg, New York, 1986. MR0851383 (88b:65129)

[19] Y. N. HE AND K. T. LI, Convergence and stability of finite element nonlinear Galerkin method for the Navier-Stokes equations, Numer. Math., 79(1998), pp. 77-107. MR:1608417 (99c:65165)

[20] J. G. Heywood and R. Rannacher, Finite element approximation of the nonstationary Navier-Stokes problem I: Regularity of solutions and second-order error estimates for spatial discretization, SIAM J. Numer. Anal., 19(1982), pp. 275-311. MR0650052 (83d:65260)

[21] J. S. LANGER, Models of patten formation in first-order phase transitions, in Directions in Condensed Matter Physics, World Science Publishers, 1986, pp. 164-186. MR0873138 (88a:82023)

[22] G. M. Lieberman, Second Order Parabolic Differential Equations, World Scientific Publishing Corp., River Edge, NJ, 1996. MR1465184 (98k:35003)

[23] F. H. Lin And C. Liu, Nonparabolic dissipative systems modeling the flow of liquid crystals, Comm. Pure Appl. Math., 48(1995), pp. 501-537. MR.1329830 (96a:35154)

[24] C. Liu And S. Shkoller, Variational phase field model for the mixture of two fluids, Preprint 2001.

[25] C. Liu AND J. Shen, A phase field model for the mixture of two incompressible fluids and its approximation by a Fourier-spectral method, Physica D, 179(2003), pp. 211-228. MR 1984386 (2004j:35233)

[26] G. B. McFadden, Phase field models of solidification, Contemporary Mathematics, 295(2002), pp. 107-145. MR1940624 (2003k:80004)

[27] R. Temam, Navier-Stokes Equations, AMS Chelsea Publishing, Providence, RI, 2001. 1846644 (2002j:76001) 
Department of Mathematics, The University of Tennessee, Knoxville, Tennessee 37996

E-mail address: xfeng@math.utk.edu

Faculty of Science, Xi'an Jiaotong University, Xi'an, Shaanxi 710049, People's RePUBLIC OF CHINA

E-mail address: heyn@mail.xjtu.edu.cn

Department of Mathematics, Penn State University, University Park, Pennsylvania 16802

E-mail address: liu@math.psu.edu 